Preprint typeset in JHEP style - HYPER VERSION

\title{
Solving matrix models using holomorphy
}

\author{
David Berenstein * \\ School of Natural Sciences, Institute for Advanced Study, Princeton, NJ 08540, USA
}

\begin{abstract}
We investigate the relationship between supersymmetric gauge theories with moduli spaces and matrix models. Particular attention is given to situations where the moduli space gets quantum corrected. These corrections are controlled by holomorphy. It is argued that these quantum deformations give rise to non-trivial relations for generalized resolvents that must hold in the associated matrix model. These relations allow to solve a sector of the associated matrix model in a similar way to a one-matrix model, by studying a curve that encodes the generalized resolvents. At the level of loop equations for the matrix model, the situations with a moduli space can sometimes be considered as a degeneration of an infinite set of linear equations, and the quantum moduli space encodes the consistency conditions for these equations to have a solution.
\end{abstract}

KEYwords: Matrix models, Supersymmetric gauge theories.

*dberens@ias.edu 


\section{Contents}

1. Introduction

2. The one matrix model 5

3. Superpotential deformations of $\mathcal{N}=4$ SUSY gauge theories 10

3.1 Calculating the classical moduli space of a probe brane.

3.2 The quantum moduli space 18

3.3 The chiral ring of the gauge field theory 20

3.4 Relations to a matrix model 22

4. Obtaining the Quantum relations from loop equations for a matrix model 26

4.1 The first equation 28

4.2 The second Quantum equation 29

5. Partial gaugino condensates 33

6. Conclusion 34

\section{Introduction}

Recent developments on supersymmetric gauge theories have shown that there is a deep relationship between SUSY theories that admit a large $N$ limit and zero dimensional matrix models [17, 18, 19]. The connection between these seemingly different physical systems arose from the study of topological string theory amplitudes for open strings, but now there are purely field-theoretic arguments that show this connection. Of the two available proofs [15, 7] of the correspondence (for a $U(N)$ gauge theory), the most compelling one was given in the paper [7], as it gave a full dictionary between loop operators on the matrix model and certain elements of the chiral ring of the gauge theory, as well as a proof that can be argued to be valid non-perturbatively. Part of the setup includes a correspondence between the gaugino condensate of the SUSY gauge theory and the 't Hooft coupling of the matrix model [19].

Having this connection makes it possible to solve for the vacuum structure of the supersymmetric gauge field theory if one knows how to solve the matrix model in the large $N$ limit. We will refer the reader to [14, 31] for reviews of matrix models. A more recent list of solvable models appears in 38

The most successful and studied example to date has been given by deforming the $U(N) \mathcal{N}=2$ gauge theory, whose solution was given by Seiberg and Witten [50] to an 
$\mathcal{N}=1$ gauge theory by adding a superpotential $\operatorname{tr}(V(X))$, where $X$ is the chiral field superpartner of the vector multiplet and $V(X)$ is a polynomial in $X$, see for example 19, 19, 13, 28, 29, 30, 32, 37, 7, 8]. This theory is related to the one matrix model with potential $V(X)$. The classical vacua of the gauge theory are described by distributing eigenvalues on the roots of the polynomial $V^{\prime}(X)$ giving rise to a theory whose classically unbroken gauge group is $\prod U\left(N_{i}\right)$, where there are $N_{i}$ eigenvalues in the $i$-th root of the polynomial $V^{\prime}(X)$.

Similarly, in the matrix model, the vacua are also described by distributing (an infinite number of) eigenvalues over the classical saddle points of the matrix model. Each of these classical points in the eigenvalue plane becomes a cut in the eigenvalue plane due to quantum effects, when one considers the spectral curve of the matrix model. For each cut there is an associated 't Hooft coupling which counts how many eigenvalues end up in the given cut. This information is related to a partial gaugino condensate [19], whose holomorphic definition was given nonperturbatively in [7].

One of the advantages of this new method of computing gauge theory results is that it does not assume S-duality like symmetries to solve the theory, so it can provide tests of these highly non-trivial symmetries. For example, aspects of the $\mathcal{N}=1^{*}$ vacuum structure have been studied in this way [23, 24], but a general solution of the vacua of the theory in terms of matrix models is not yet available. ${ }^{1}$

Part of the failure to obtain the full solution of the matrix model comes from the fact that the classical $\mathcal{N}=1^{*}$ gauge theory vacua is described by a decomposition into irreducible representations of $S U(2)$, and there are an infinite number of such representations. In the associated matrix model, this translates into an infinite number of classical saddle points for the eigenvalues of one of the matrices, let us call it $X$, and to each such classical saddle point for the eigenvalue $x$ of $X$ one can associate a cut in a spectral curve of the quantum theory (at least, this is what experience dictates from the one-matrix model). In particular, one has a potentially infinite number of cuts. This suggests that one can not solve the loop equations of the matrix model in a straightforward fashion, as there will be an infinite number of unknown parameters: one can take these parameters to be the number of eigenvalues in each cut $N_{i}$, but more to the point, the one point functions $<X^{n}>$ depend classically on all of the $N_{i}$. Thus, there are no recursion relations which solve for the one point functions of the loop operators $\left\langle X^{n}\right\rangle$.

The technical issue is how to effectively truncate the problem to finitely many cuts. In the one matrix model this is automatic, but in the $\mathcal{N}=1^{*}$ theory one has to do this by hand. A related problem in complex analysis can be phrased as follows: given a Laurent series of an analytic function $f(z)=\sum a_{i} z^{-i}$ at infinity, what are the constraints on the variables $a_{i}$ that guarantee that $f(z)$ has a finite number of cuts in the interior of the complex plane? The constraints involve an infinite number of the coefficients of $f$ at the same time, since multiplying $f(z)$ by any polynomial function of $z$ will always give a

\footnotetext{
${ }^{1}$ This theory can also be solved by first turning on a mass term deformation that gives rise to an $\mathcal{N}=2$ gauge theory, and finding the associated integrable system [21, 22, 36]. In principle, it is possible to evaluate all of the holomorphic information by going to the appropriate point in the moduli space of vacua.
} 
function with the same properties. Looking at it from this point of view it also looks like the problem has no solution.

The current literature solves the abstract problem by making a clever anzatz for the one-cut solution (or multi-cut solutions where all of the cuts are correlated, see for example [39, 23, 24, 25, 45]), that leads to studying functions on an elliptic curve, but it does not solve in any sense a general multi-cut solution.

In this paper we will describe a new technique to solve (only partially) similar matrix models based on getting information from the gauge theory first. Since the gauge theory and the matrix model data are in some sense equivalent, this statement might seem paradoxical. We will concern ourselves with situations where the superpotential is non-generic and there is the possibility of having a moduli space of vacua. In particular, the allowed deformations of the moduli space give a finite number of parameters which determine part of the vacuum structure. In the matrix model these turn out to be situations where the system of loop equations is degenerate in some sense, a statement which will be explained in detail later in the paper, and there are more free parameters than at a generic case. The main question we want to address is: what does the moduli space tell us about the matrix model?

To avoid undue suspense, we find that under some circumstances the existence of a moduli space of vacua produces an integrable sector of the matrix model, this is, part of the problem behaves like a one matrix model: one can find an infinite number of one point functions with a finite amount of data. The finite amount of data is exactly the one that characterizes the possible deformations of the moduli space that are allowed by holomorphy.

To show the new technique, we will study two examples. First, a toy example which reduces to the one matrix model, which we understand very well. It will just be an illustration of the matrix model techniques used. Then we will study a three matrix model closely related to the $\mathcal{N}=1^{*}$ gauge theory which has special properties that insure that certain objects in the matrix model only have a finite number of cuts automatically. This happy coincidence is tied to the fact that these particularly gauge theories can be geometrically engineered. We will in this paper always consider a gauge field theory which can be geometrically engineered by placing branes on a singular Calabi-Yau (CY) geometry. From this point of view we are exploring questions that relate the CY geometry to a matrix model.

The basic idea is that if we place fractional branes at the singularities of the geometry, then the CY geometry will be deformed by geometric transitions so that the singularities with fractional branes on them are 'resolved'. This will be exactly like in the KlebanovStrassler geometry [40, 33], repeated many times at many singularities, as in [12, 10].

If we insert a probe brane in the bulk (in the presence of the fractional branes) then it's moduli space should correspond to the deformed CY geometry. This idea is very common in the literature, see for example [40, 34, 35, 44. Fortunately, the shape of the CY can be studied directly from the field theory [6, 3] so it is possible to start with a classical gauge field theory and produce certain CY geometries, as opposed to start with the geometry and guess the field theory. If we place a brane in the bulk and compute it's moduli space, the 
moduli space of the brane will be the CY geometry. Of course, not every superpotential will lead to a CY geometry, but for those that do one can make progress in understanding the gauge theory questions by studying the geometry (moduli space of the theory). Also, one should be able to understand aspects of the matrix model by studying the geometry.

The main advantage with this setup is that the problem is controlled by holomorphy: the possible deformations of the CY geometry that result from placing fractional branes at singularities are not arbitrary, so there are only a finite number of unknowns. This is exactly what we are looking for, a situation where the potentially infinite number of variables (cuts in the matrix model) is reduced to finitely many (we are assuming that there is one cut per quantum deformation parameter, a statement which will be justified later).

We want to study exactly what matrix model information we can get from the gauge theory moduli space. We will use a proposal first presented in [ [ ] to calculate the quantum moduli space from the matrix model. The relation between the moduli space and how to obtain it from the matrix model still lacks a proof, so it should be treated as a conjecture that needs justification. However, one can make predictions based on the calculation of the deformed moduli space from the matrix model, and the one expected from holomorphy. Some of these predictions can be verified directly by manipulating the loop equations of the matrix model, and we will use this calculation as a consistency check of the proposal.

In our examples, we will not solve the matrix model completely (find all one point functions from a finite amount of data). We will use the word 'solve' in the following more restricted sense: it is possible to determine an infinite number of one point functions of the matrix model with a finite amount of data. In essence, we obtain similar results to a one matrix model.

The main example we will study is a gauge theory that will have three matrices $X, Y, Z$ in the adjoint of $U(N)$, and a superpotential of the general form

$$
\operatorname{tr}(X Y Z-q X Z Y+V(X))
$$

with the restriction $q^{n}=1$, and a restriction on the form of $V(X)$ which depends on $n$. Similar systems have been studied in [26, 27, 5, 25, 36, 45] and are interesting on their own right as they encode structure of interesting deformations of $\mathcal{N}=4$ gauge theories [44. The restrictions placed on the superpotential guarantee that there is a CY geometry associated to the gauge theory [26, 27, 5], and similar examples studied in [12, 11]. Also, after a linear change of variables on $X$, we can get for $q \neq 1$ an equivalent superpotential of the form

$$
\operatorname{tr}\left(X Y Z-q X Z Y+\tilde{V}(X)+m^{2} Y Z\right)
$$

The $\mathcal{N}=1^{*}$ gauge theory has the same matter content and the potential

$$
\operatorname{tr}\left(X Y Z-X Z Y+\tilde{m}^{2} X^{2}+m^{2} Y Z\right)
$$

so by varying $q$ we can in principle get arbitrarily close to the $\mathcal{N}=1^{*}$ gauge theory. This particular model has been studied extensively, also in relation to AdS/CFT [46, 1]. This is the main physical motivation to write this paper: to approach the problem of solving the 
$\mathcal{N}=1^{*}$ vacuum structure from the matrix models, with an arbitrary number of cuts, as well as the related $q$ deformed systems. It is important to notice that setting $q \neq 1$ means that the conformal field theory with $V=0$ has only $N=1$ SUSY in four dimensions. Therefore, techniques that depend on the quantum corrections being calculable in $\mathcal{N}=2$ theories, which then are softly broken to $\mathcal{N}=1$ can break down because there is less supersymmetry protecting the system. In particular, to use integrable systems like in $\mathcal{N}=2$ gauge theories, but with less supersymmetry [36], one actually needs a proof that the moduli space does not get lifted by quantum corrections. These matrix models are also interesting on their own right, see for example [43], so we can also gain insight into the problem of solving matrix models by using four dimensional SUSY gauge theory information.

\section{The one matrix model}

In this short section we will show how the analysis of a quantum moduli space can solve the one matrix model. The whole purpose of this section is to show that a quantum deformed CY geometry contains information about loop equations of the matrix model.

Consider the CY threefold geometry

$$
u v=(w-P(z))(w+P(z))
$$

where $P(z)$ is a polynomial of degree $n\left(P(z)=\sum_{i=0}^{n} a_{i} z^{i}\right)$, and which results from deforming the $A_{1}$ singularity geometry $u v=w^{2}$. This procedure removes the codimension two singularities and leaves only codimension three singularities behind, which for generic $P(z)$ are conifold singularities.

The gauge theory associated to D-branes on this geometry has been studied in [12, 11, 10, 9], and it is given by a quiver diagram with two nodes, representing the affine dynkin diagram $\hat{A}_{1}$.

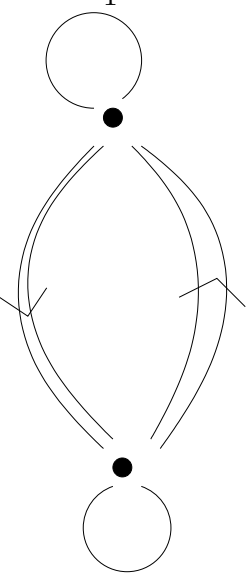

Because there are two nodes in the quiver theory, one has general gauge group $U(N) \times$ $U(M)$. The superpotential of the theory is given by

$$
W=\operatorname{tr}\left(Z A_{1} B_{1}-B_{1} A_{1} \tilde{Z}-Z A_{2} B_{2}+B_{2} A_{2} \tilde{Z}\right)+\operatorname{tr}(V(Z)-V(\tilde{Z})
$$

and in the case $V(x)=0$ the superpotential is the one that results from an $\mathcal{N}=2$ supersymmetric gauge theory. $P(x)$ as a polynomial is determined by the following equation 
$2 P(x)=V^{\prime}(x)$, so one can relate the shape of the geometry and the gauge theory parameters in the superpotential.

A brane in the bulk will have a theory characterized by the gauge group $U(1) \times U(1)$. This is the correct fractional brane content for a bulk brane in the orbifold geometry, and these numbers do not change when we deform the geometry.

Also, if we take $M=0$, we see that we obtain a gauge theory with the same matter content as the $\mathcal{N}=2, U(N)$ gauge theory, which has been deformed by the potential $V(Z)$. Thus this particular theory is a sub-theory of the one defined by the geometry.

This $U(N)$ does not have a moduli space of vacua, instead it has a discrete set of vacua. These vacua at the classical level are built by distributing the $N$ eigenvalues of $Z$ into the roots of $P(Z)$. If there are $N_{i}$ eigenvalues at the $r_{i}$ root, the low energy effective (classical) theory is given by pure gauge theory, with gauge group $\prod_{i} U\left(N_{i}\right)$. Quantum effects lead to confinement of the $S U\left(N_{i}\right)$ groups, so in the infrared of the quantum theory we end up with a gauge group $\prod(U(1))$ over the roots which have at least one eigenvalue.

To solve for the structure of the vacuum, we want to find the vevs of all of the elements of the chiral ring. The chiral ring is generated by the following combinations

$$
\begin{array}{r}
\frac{1}{32 \pi^{2}} \operatorname{tr}\left(Z^{n} W_{\alpha} W^{\alpha}\right) \\
\operatorname{tr}\left(Z^{n} W_{\alpha}\right) \\
\operatorname{tr}\left(Z^{n}\right)
\end{array}
$$

The ordering of $W$ inside the trace does not matter, because any other ordering can be obtained by the addition of terms of the form $\bar{D} M$, so at the level of the chiral ring they give the same cohomology class. For our purposes, the elements of the chiral ring which have a simple interpretation in a matrix model are the ones given by $\operatorname{tr}\left(Z^{n} W_{\alpha} W^{\alpha}\right)$. Using the generalized Konishi anomaly for the variations $\delta Z \sim Z^{k} W_{\alpha} W^{\alpha}$, Cachazo et al. [7] were able to show that these satisfy the same equations as the loop equations for the one matrix model with potential $V(X)$. This is

$$
\operatorname{tr}\left(V^{\prime}(Z) Z^{k} W_{\alpha} W^{\alpha}\right)=\frac{1}{32 \pi^{2}} \sum\left(\operatorname{tr}\left(Z^{k-1-i} W_{\alpha} W^{\alpha}\right) \operatorname{tr}\left(Z^{i} W_{\alpha} W^{\alpha}\right)\right.
$$

If we call $O_{i}=\frac{1}{32 \pi^{2}} \operatorname{tr}\left(Z^{i} W_{\alpha} W^{\alpha}\right)$, then the equations above read

$$
\operatorname{tr}\left(\sum a_{j} Z^{k+j} W_{\alpha} W^{\alpha}\right)=\frac{1}{32 \pi^{2}} \sum\left(\operatorname{tr}\left(Z^{k-1-i} W_{\alpha} W^{\alpha}\right) \operatorname{tr}\left(Z^{i} W_{\alpha} W^{\alpha}\right)\right.
$$

or equivalently

$$
\sum a_{j} O_{k+j}=\sum_{i} O_{k-i-1} O_{i}
$$

This equation can be read as a recursion relation for the vevs $O_{n+k}$ for $k \geq 0$ in terms of the vevs $O_{0}, \ldots, O_{n-1}$. All of these equations can be put in one single equation in terms of a generating function for the $O_{i}$, defined as follows

$$
R(u)=\sum O_{k} u^{-k-1}
$$


so that

$$
V^{\prime}(u) R(u)=R(u)^{2}+f(u)
$$

where $f$ is an unknown polynomial of degree $n-1$. This is necessary to seed the initial conditions for the recursion relation. Given $f$ it straightforward to solve for $R(u)$. $f$ in the quantum theory is determined by how we choose to distribute the eigenvalues of $Z$ on the different roots of the classical potential, and it encodes the different possible vacua of the theory.

These equations above are the same equations that can be derived from a one matrix model with potential $V(X)$. This is, we have the matrix model

$$
\int[d X] \exp \left(-N \mu^{-1} \operatorname{tr}(V(X))\right)
$$

which is to be solved in the planar limit in the large $N$ limit. We can write the loop operators

$$
<X^{k}>=\frac{1}{N} \operatorname{tr}\left(X^{k}\right)
$$

and the loop equations for the one matrix model

$$
<V^{\prime}(X) X^{k}>=\mu \sum_{i}<X^{k-1-i}><X^{i}>
$$

So it is immediate to see that they have structurally the same form as the ones given above (by setting $O_{i} \sim \mu<X^{i}>$ ). In the matrix model we have the normalization condition $<X^{0}>=<1>=1$, which identifies $\mu \sim \frac{1}{32 \pi^{2}}<W_{\alpha} W^{\alpha}>=S$, the gaugino condensate. It is the identification between loop equations of a matrix model and the chiral ring constraints that allow us to relate these two very different mathematical problems.

Now, let us use the quantum moduli space technique to rederive the same loop equations from a different point of view. The idea is to study the gauge theory with potential 2.2, with one brane in the bulk, this is, with gauge group $U(N+1) \times U(1)$.

The classical moduli space is obtained by solving the F-term constraints. The constraints for the $A, B$ give us

$$
z A_{i}-A_{i} \tilde{z}=0 \quad B_{i} z-\tilde{z} B_{i}=0
$$

and since $\tilde{z}$ is a scalar, for $A, B$ to get a non-zero vev and give us a brane in the bulk, we need that one of the eigenvalues of $z$ is equal to $\tilde{z}$, and that $A, B$ be eigenvectors of the matrix $z$ with eigenvalue $\tilde{z}$.

We can diagonalize $z$, and single out the $(1 \times 1)$ block matrices corresponding to the eigenvalue $\tilde{z}$. With this convention

$$
z=\operatorname{diag}\left(\tilde{z}, z_{1}, \ldots z_{n}\right), \quad B=\left(b_{i}, 0, \ldots, 0\right) \quad A_{i}=\left(\begin{array}{c}
a_{i} \\
0 \\
\vdots \\
0
\end{array}\right)
$$


The other eigenvalues will be constrained by the equations

$$
A_{1} B_{1}-A_{2} B_{2}=V^{\prime}(z) \quad B_{1} A_{1}-B_{2} A_{2}=V^{\prime}(\tilde{z})
$$

which result in $V^{\prime}\left(z_{i}\right)=0$ and

$$
b_{1} a_{1}-b_{2} a_{2}=V^{\prime}(\tilde{z})
$$

From here, the classical eigenvalues $z_{i}$ which were not singled out will be distributed only along the saddle points of the potential $V$.

Now, to find the moduli space we need to write the constraints between the gauge invariant variables (with respect to the group $U(N+1)$ ) given by $\tilde{z}$ and the combinations

$$
u=a_{1} b_{2}, v=a_{2} b_{1}, w=\frac{a_{1} b_{1}+a_{2} b_{2}}{2}, \Omega=\frac{a_{1} b_{1}-a_{2} b_{2}}{2}
$$

Clearly, the F-terms imply $\Omega=V^{\prime}(\tilde{z}) / 2$. And we can also use the algebraic relation

$$
w^{2}=\Omega^{2}+u v=\frac{V^{\prime}(\tilde{z})^{2}}{4}+u v
$$

to obtain the equations describing the moduli space given by the geometry 2.1.

The classical moduli space is given by the single equation in four variables $w, u, v, \tilde{z}$

$$
w^{2}-u v-\frac{V^{\prime}(\tilde{z})^{2}}{4}=0
$$

and it has branes located at the singularities of the geometry. Because there are singularities, it is possible to deform the moduli space and remove the singularities. These deformations are controlled by holomorphy.

We should expect that the quantum deformed moduli space is of the form

$$
w^{2}-u v-\frac{V^{\prime}(\tilde{z})^{2}}{4}=\text { Quantum deformations }=Q
$$

where the right hand side is a polynomial involving only positive powers of the confining scale $\Lambda_{S U(N+1)}$, and which is polynomial in the coefficients of $P$. This is the expected result from holomorphy [47, 48, 49].

The particular theory at hand has an $S U(2)$ symmetry under which $A_{1}, A_{2}$ and $B_{1}, B_{2}$ are doublets, while $z, \tilde{z}$ are singlets. $w, u, v$ together form a triplet, and $\Omega$ is a singlet. From these symmetries one can see that the quantum deformations will be independent of $w, u, v$, since terms of degree one in these variables that could appear in the polynomial $Q$ above are not in a singlet representation of $S U(2)$. Hence $Q$ can only be given by a polynomial in $z$. The deformations should also be such that they are subleading with respect to the deformations induced by changing the couplings in the superpotential. If $V^{\prime}(z)$ is of degree $n$, and we change the functional form of $V^{\prime}$ we can vary the coefficients of $z^{n}, z^{n+1}, \ldots, z^{2 n}$ independently of each other. The deformation should thus be of the form $f(z)$, with $f$ of degree smaller than $n$ [12].

It turns out that the number of parameters in the deformation $f$ is equal to the number of roots of $V^{\prime}$. For each of these roots there is a conifold singularity, and each of these 
singularities can be deformed away by placing fractional branes at the given conifold, thus inducing a geometric transition which is of the same type as the one in 40]. We get in the end the same number of deformation parameters as there are singularities in the geometry.

Now we want to see that these deformations can be related to the loop equations of the matrix model with potential $V$.

For this, we need to know how to derive the quantum moduli space of the gauge field theory from the matrix model point of view. This has been previously argued for in 何.

We need to consider a multi-matrix model with the same field content as the gauge theory, and we take $\tilde{N} \rightarrow \infty$. However, we fix the matrix model so that the number of moduli stays fixed. This is, we will consider a matrix model with one probe brane in the bulk singled out. We do this with a matrix model potential given by the classical superpotential of the theory.

$$
\int([d z][d \tilde{z}][d A][d B])^{\prime} \exp (W)
$$

where the prime indicates that we leave some matrix fields unintegrated (exactly those that are massless perturbatively in the gauge theory). Since in the matrix model we do a large $N$ expansion, we are going to get to the results by analyzing the saddle point equations for the variables $\tilde{z}$, and what we called $a_{i}, b_{i}$ perturbatively above. Also, one of the eigenvalues of $z$ in the gauge theory is singled out to be equal to $\tilde{z}$ perturbatively. This eigenvalue will be called $z_{0}$ in the matrix model. At least naively, we can ignore the effect of the matrix probe on the large $N$ matrix condensate because it would give rise to a $1 / N$ effect.

On going to a basis of eigenvalues for $z$, and including the Vandermonde determinant we find the following integral to perform in the large $\tilde{N}$ limit:

$$
\begin{gathered}
\quad \int \prod_{i \neq 0}\left[d \lambda_{i}\right]\left[d A_{12}^{i}\right]\left[\left(d B_{12}\right)_{i}\right] \Delta^{2} \exp -\left\{\tilde{N} \mu^{-1} \sum_{j=1}^{\tilde{N}}\left(\lambda_{j}-\tilde{z}\right)\left(A^{j} \cdot B_{j}\right)+\right. \\
\left.+\tilde{N} \mu^{-1} \sum_{j=0}^{\tilde{N}} V\left(\lambda_{j}\right)-\tilde{N} \mu^{-1} V(\tilde{z})+\tilde{N} \mu^{-1}\left(z_{0}-\tilde{z}\right)\left(\left(a_{1}\right)\left(b_{1}\right)-\left(a_{2}\right)\left(b_{2}\right)\right)\right\}
\end{gathered}
$$

The above equation includes the Vandermode for all of the eigenvalues of $z$, not just the Vandermonde of the large $N$ condensate. In the equations above $\Delta^{2}$ is the Vadermonde determinant. The sum over $j$ of $A^{j} B_{j}$ is over the color indices associated to the eigenvalues $\lambda_{1}, \ldots \lambda_{\tilde{N}}$, but not to $z_{0}$. This has been separated in the last line because we want $a_{1}, b_{1}, a_{2}, b_{2}$ to be moduli in the matrix model, so they cannot be massive at the saddle point. From this condition one of the eigenvalues of $z$ is equal to $\tilde{z}$.

In the equation above it is possible to integrate $A^{j}, B_{j}$ completely. This being a Gaussian integral over four coordinates of mass $\lambda_{j}-\tilde{z}$ gives us a measure term equal to $\delta=\prod_{i \neq 0}\left(\lambda_{i}-\tilde{z}\right)^{-2}$

The logarithm of the Vandermonde determinant combined with $\delta$ is then

$$
\log \left(\Delta^{2} \delta\right)=\sum_{i \neq j} 2 \log \left(\lambda_{i}-\lambda_{j}\right)-2 \sum_{i \neq 0} \log \left(\lambda_{i}-\tilde{z}\right)
$$

and it is the term in the effective action for the eigenvalues $\lambda$ and $z$. Now we want to solve for the saddle point of this setup in the large $\tilde{N}$ limit. This will result in summing all of the 
planar diagrams of massive fields in the above theory. Notice that the eigenvalues $\tilde{z}$ and $z_{0}$ do not have an interaction between them, because we have not integrated out the massless modes of $A, B$. The saddle point equation for the zero components of $A, B$ generic make $\tilde{z}=z_{0}$ in this situation.

One sees that the saddle point equations for the eigenvalues $\lambda_{i}$ are the same as when we have the theory $U(\tilde{N}) \times U(0)$, because the contribution from $z_{0}$ cancels the contribution from $z$ when they are equal. This seems accidental in the matrix model. The saddle point equation for the eigenvalues $\lambda_{i}$ is

$$
\tilde{N} \mu^{-1} V^{\prime}\left(\lambda_{i}\right)-2 \sum_{j \neq i} \frac{1}{\lambda_{i}-\lambda_{j}}=0
$$

Now let $w(\lambda)=\frac{1}{\tilde{N}} \sum_{i \neq 0} \frac{1}{\lambda-\lambda_{i}}$ be the resolvent of the matrix model.

The saddle point equations for $\tilde{z}=z_{0}$ are identically equal to

$$
V^{\prime}(\tilde{z})-2 \mu w(\tilde{z})+a_{1} b_{1}-a_{2} b_{2}=0
$$

From here, we can use the same identity that led us to the classical moduli space to find that the quantum moduli space, as described by the matrix model, should be given by the equation

$$
w^{2}-u v=\frac{1}{4}\left(V^{\prime}(\tilde{z})-2 \mu w(\tilde{z})\right)^{2}
$$

Now, we compare the matrix model prediction to the prediction obtained by holomorphy arguments. It should be the case that two different functions of $\tilde{z}$ are identical, these are as follows

$$
\frac{1}{4}\left(V^{\prime}(\tilde{z})-2 \mu w(\tilde{z})\right)^{2}=\frac{1}{4}\left(V^{\prime}(\tilde{z})\right)^{2}+f(z)
$$

where $f$ is a polynomial. We obtain from these the following set of equations (after changing the normalization of $f$ to $\tilde{f}=-4 \mu^{-1} f$ )

$$
V^{\prime}(\tilde{z}) w(\tilde{z})-\mu w(\tilde{z})^{2}=\tilde{f}(\tilde{z})
$$

which encode the full set of loop equations of the one matrix model 2.10, when we realize that $R(u)$ and $w(\tilde{z})$ are really the same object.

To summarize: the quantum deformed moduli space of a supersymmetric gauge theory can encode loop equations for a matrix model. The above result for a one matrix model is not new and seems redundant, but the results in the following sections, using the same techniques are new.

\section{Superpotential deformations of $\mathcal{N}=4 \mathrm{SUSY}$ gauge theories}

The $\mathcal{N}=4$ SUSY gauge theory is a supersymmetric field theory characterized by a gauge group $G^{2}$ and three fields $X, Y, Z$ in the adjoint whose superpotential (up to normalization) is given by

$$
\operatorname{tr}(X Y Z-X Z Y)
$$

\footnotetext{
${ }^{2}$ The gauge group will be $U(N)$ for the remainder of the paper. The results presented here can be generalized to other gauge groups which admit a large $N$ description
} 
This theory is a superconformal field theory which spontaneously breaks the conformal invariance at generic points in the moduli space, but if the vacuum is at the origin in moduli space (the gauge group is unbroken) then the theory has a vacuum with superconformal invariance.

The gauge theory with $\mathcal{N}=4$ SUSY has a moduli space of conformal field theories parametrized by the gauge coupling of the theory $g$. If we consider deformations of the superpotential, this moduli space of $\mathcal{N}=1$ superconformal field theories possesses a three parameter family of deformations which give rise to superconformal field theories [44]. These have (up to normalization) the following form of the superpotential

$$
W_{0}=\operatorname{tr}\left(X Y Z-q X Z Y+\frac{\beta}{3}\left(X^{3}+Y^{2}+Z^{3}\right)\right)
$$

and they also respect a $\mathbb{Z}_{3}$ symmetry which permutes $X \rightarrow Y \rightarrow Z$. When $\beta=0$ on top of the $U(1)_{R}$ symmetry, there is an additional non-anomalous $U(1)^{2}$ global symmetry of rotations of $X, Y, Z$ by phases.

It has been shown that some of these can have very interesting brane descriptions and $A d S / C F T$ geometric duals [26, 5], of the form of branes on $\mathbb{C}^{3} /\left(\mathbb{Z}_{n} \times \mathbb{Z}_{n}\right)$ or $A d S_{5} \times$ $S^{5} /\left(\mathbb{Z}_{n} \times \mathbb{Z}_{n}\right)$ for $\beta=0$ and $q^{n}=1$. These are geometric theories with branes on orbifold singularities, and there are three lines of singularities in codimension two that extend to infinity (one can think of them as the $X, Y, Z$ axis). From here one finds a host of deformations of the geometry which serve to remove the codimension two singularities, leaving only codimension three singularities behind ${ }^{3}$.

Since these examples are noncompact Calabi-Yau manifolds, one has to take care in defining the set of allowable deformations that one can study, as the total number of holomorphic functions describing the deformations is infinite. The deformations of the geometry modify the superpotential of the brane, so it is possible to analyze the geometry given a superpotential.

For the rest of the paper we will consider only a special form of the general superpotential described above:

$$
W=\operatorname{tr}(X Y Z-q X Z Y+V(X))
$$

Where $q^{n}=1$ and

$$
V(x)=\sum_{i \neq 0} \frac{a_{i}}{i} x^{i}
$$

is a polynomial potential.

The first condition guarantees that there is a non-anomalous $U(1)_{Y Z}$ symmetry under which $Y, Z$ have opposite charges, and is a remnant of the $S U(4)$ R-symmetry of the $\mathcal{N}=4$ gauge theory. This symmetry serves to simplify the problem. ${ }^{4}$

\footnotetext{
${ }^{3}$ These codimension three singularities are usually of the conifold type, see the examples in [27, 2]

${ }^{4}$ One could also study by the methods presented here a situation where $W=\operatorname{tr}(X Y Z-q X Z Y+V(X)+$ $\left.m\left(Z^{2}+Y^{2}\right)+\alpha Z+\alpha^{\prime} Y\right)$ but this example requires a lot more algebra to understand. the one cut solution has been studied in 45
} 
The second point to notice is that when the $a_{i}=0$ we can use the other $U(1)$ charge combined with the $R$ symmetry in such a way that the field $X$ has different charge than $Y, Z$ and we can also choose it in such a way that $X, Y, Z$ have all positive charge as well as all of the coefficients in $V(X)$, when we introduce them and assign quantum numbers to the $a_{i}$ so that we keep the global symmetry. Let us call this charge $U(1)_{R^{\prime}}$

From this point of view, the deformation described above is a relevant deformation of the gauge field theory (when considering the grading of potential terms given by the charge), because if we consider the coefficients as ordinary c-numbers, the $R^{\prime}$ charge of the deformation is smaller than the charge of the original superpotential.

The second condition given by equation 3.4 is more tricky to understand. From the string theory point of view it amounts to the deformations of the geometry being given exclusively by turning fields in the twisted sector of the orbifold which have charge zero under the $U(1)_{Y Z}$ symmetry. These guarantee that the dimension of the moduli space of a brane in the bulk remains the same as in the theory without the deformation, namely, the deformation can be understood geometrically and it does not produce an effective potential on the moduli space. In what follows we will argue what form the moduli space takes when we turn these deformations on.

\subsection{Calculating the classical moduli space of a probe brane.}

As a supersymmetric problem, we need to solve the F-term constraints resulting from the classical superpotential as described above. These classical equations that determine the vacua are given by

$$
\begin{aligned}
& X Y-q Y X=0 \\
& Z X-q X Z=0 \\
& Y Z-q Z Y=-V^{\prime}(X)=-\sum_{i \neq 0} a_{\bmod (n)} a_{i} X^{i-1}
\end{aligned}
$$

and for a probe brane in the bulk the rank of the group $N$ turns out to be equal to $n$ [26].

In particular if we think of $X, Y, Z$ as formal symbols instead of matrices, we obtain the result that finding solutions to the equations above in terms of matrices is the same problem as finding representations of an associative algebra over the complex numbers, generated by $X, Y, Z$, and subject to the relations [3.5 [5, 6], which are exactly of dimension $n$. We are particularly interested in irreducible representations of the above algebra of dimension $n$, because this is the rank associated to a brane in the bulk. The irreducibility implies that the gauge group is broken to $U(1)$ in the infrared, which is the gauge group associated to a single brane in the bulk.

Finding these representations will let us calculate the classical moduli space of the theory and recover the Calabi-Yau geometry. The holomorphic functions on the CalabiYau geometry will be given by functions which are in the center of the above algebra [5, 6, 3]; and the relations between these functions will be the relations that follow by virtue of imposing the equations 3.5. In an irreducible representation all of these variables in the center can be shown to be proportional to the identity due to Schur's lemma. Hence 
these are essentially gauge invariant, as they can be recovered completely from their trace. From this point of view, the relations in the center of the algebra are relations in the classical chiral ring of a one probe brane system.

We need to solve the problem of finding the set of irreducible representations of dimension $n$ of the above algebra and the center of the algebra. To do this we need to solve for the F-terms above in dimension $n$.

Solving the first equation is easy if we introduce the clock and shift matrices

$$
P=\left(\begin{array}{ccccc}
1 & & & \\
& & & & \\
& & & & \\
& q^{2} & & \\
& & \ddots & \\
& & & \\
& & & q^{n-1}
\end{array}\right), Q=\left(\begin{array}{ccccc}
0 & 1 & 0 & \ldots & 0 \\
0 & 0 & 1 & \ldots & 0 \\
\vdots & \vdots & \vdots & \ddots & 0 \\
0 & 0 & 0 & \ldots & 1 \\
1 & 0 & 0 & \ldots & 0
\end{array}\right)
$$

which satisfy $Q P=q P Q, Q^{n}=P^{n}=1$. These two matrices are linearly independent and generate the matrix ring of $n \times n$ matrices. A general $n \times n$ matrix $M$ can be written in a unique form as a linear combination $M=\sum_{i, j=0}^{n-1} a_{i j} P^{i} Q^{j}$.

One can show that the first equation in 3.5 is solved (generically) up to equivalence by $X=x P$ and $Y=y Q$. This is the case when $Y^{n}=y^{n} \neq 0$ and $X^{n}=x^{n} \neq 0$. To solve the second equation, the most general solution has to be of the form

$$
Z=z Q^{-1} P^{-1}+\sum_{i=1}^{n-1} z_{i} Q^{-1} P^{i-1}
$$

If we now substitute this expression in the third equation, we obtain that

$$
\sum y z_{i} P^{i-1}\left(1-q^{i}\right)=-\sum a_{i} x^{i-1} P^{i-1}
$$

Since the powers $P^{i}$ are linearly independent, we can solve for the $z_{i}$ as follows

$$
z_{i}=-a_{i} y^{-1} x^{i-1} /\left(1-q^{i}\right)
$$

Thus we find that the generic irreducible representations of dimension $n$ are parametrized by three variables $x, y, z$. However, one can show that various values of $x, y, z$ can correspond to the same irreducible representation. The equivalent classes can be distinguished by evaluating the coordinates in the center of the algebra. Notice that the equation 3.8 can only be solved if no term proportional to $P^{-1}$ appears on the right hand side of the equation. This is exactly the restriction that was imposed on the form of the superpotential.

We have found above a three-parameter family of irreducible representations. However, gauge invariance makes it possible to change $x \rightarrow q x$ with some other modifications on $y$ and $z$, so the variables above describe a cover of the moduli space. We need to evaluate gauge invariant combinations of $x, y, z$.

It is easy to show that $u=X^{n}=x^{n}$ is in the center of the algebra. From the representation above it is also easy to show that $v=Y^{n}=y^{n}$ is proportional to the identity in the representation. Since $Y, Z$ appear essentially on the same footing in the 
equations 3.5, then it follows that $w=Z^{n}$ is also in the center; although it is difficult to write an expression for $Z^{n}$ in terms of the $a_{i}, x, y, z$. Finally, there is another variable in the center, which we will call $t$ and whose form is given by $t=X Y Z+f(X)$, where $f$ a polynomial. $X Y Z$ can be readily calculated in the representation above to give

$$
X Y Z=x y z+\sum_{i=1}^{n-1} z_{i} y x P^{i}=x y z-\sum a_{i}\left(1-q^{i}\right)^{-1} x^{i} P^{i}=t-f(X)
$$

so that $f(X)=\sum a_{i}\left(1-q^{i}\right)^{-1} X^{i}$, and $t=x y z$.

We see that in this particular matrix representation $u, v, t$ are easy to calculate, and $w$ is hard to calculate. Also, these are four variables determined algebraically by three parameters $x, y, z$, so they must satisfy some algebraic relation.

In the particular case where $V^{\prime}(X)=0$, one easily finds that $w=Z^{n}=z^{n}$, and then the variables $u, v, w, t$ satisfy the following equation

$$
u v w=t^{n}
$$

With the addition of $V$, the relation above is deformed. The importance of the calculation done above was to find the expression that described the variable $t$.

The classically deformed relation above is relatively easy to calculate if we choose to write $Z$ in a slightly different form, so that $w$ can be read more easily

$$
Z=\left(\begin{array}{ccccc}
0 & & & & \zeta_{0} \\
\zeta_{1} & 0 & & & \\
& \zeta_{2} & 0 & & \\
& & \ddots & \ddots & \\
& & & \zeta_{n-1} & 0
\end{array}\right)
$$

Then we find that $Z^{n}=\prod \zeta_{i}$, and we can write equations for the $\zeta_{i}$ in terms of $t$. Namely

$$
X Y Z=x y\left(\begin{array}{cccc}
\zeta_{0} & & & \\
& \zeta_{1} & & \\
& & \ddots & \\
& & & \zeta_{n}
\end{array}\right)=t-f(X)
$$

which reduce to $x y \zeta_{i}=t-f\left(q^{i} x\right)$. Taking the product of all of these equations we find

$$
x^{n} y^{n} \prod \zeta_{i}=u v w=\prod\left(t-f\left(q^{i} x\right)\right)
$$

Now, because the right hand side is invariant when we change $x \rightarrow q x$, the right hand side only depends on $u=x^{n}$ and $t$. So we find that the deformed geometry corresponding to the change in the superpotential generated by $V$ is the following hypersurface in four complex variables $u, v, w, t$

$$
u v w=Q(t, u)
$$

where $Q$ is a polynomial in $t$ and $u$ of order $n$ in $t$ and of the same order as $V^{\prime}$ in $u$. 
One can also notice that the polynomial $Q(t, u)$ is not a generic polynomial in $t, u$ of the given order, but it depends on fewer parameters (only the variables $a_{i}$ ).

When this happens, the geometry described above has some special properties that make it non-generic. In the above case, one can expect that the non-genericity of $Q(t, u)$ is related to the existence of singularities for the curve in the $u, t$ variables described by $Q(t, u)=0$. The singularities of the CY geometry can be obtained by demanding that the partial derivative with respect to the variables $u, v, w, t$ of the hypersurface equation vanish.

The derivatives with respect to $v, w$ give rise to the following equations

$$
u v=u w=0
$$

These can be solved for general $u \neq 0$ by $v=w=0$. Under these conditions the CY has singularities at some values of $t, u$ for which the curve $Q(t, u)=0$ is singular. These singularities are in general of the conifold type. These are easy to find: just look for repeated roots of $t$. These are values of $x$ such that $f\left(q^{r} x\right)=f(x)$ for some $r \neq 0$.

A second set of singularities is produced by setting $u=0$. In this case it is easy to show that $Q(t, 0)=t^{n}$, and for $n \neq 1$ the derivative of $Q$ with respect to $t$ vanishes at $t=0$. This produces a curve of singularities characterized by the equation $v w=\left.Q_{u}(t, u)\right|_{u=0}$. This curve degenerates to two of the three lines of singularities that are present in the orbifold geometry when $Q_{u}(t, 0)=0$. This is a curve of $A_{n-1}$ singularities.

The singularities which will be important for us are the isolated singularities that we described first, and which are of the conifold type. At a conifold singularity there are usually two types of fractional branes present (even in the case where the singularities seem to have discrete torsion [2]), which wrap the local cycle of zero geometric size with the two possible different orientations.

The fractional branes in the above situation are obtained by taking a brane in the bulk to a singularity. The brane representation then becomes reducible and can be written as a direct sum of two sub-representations $\lim R=R_{\alpha}^{+}, R_{\alpha}^{-}$. Each of these sub-representations behaves in the classical low energy theory exactly as in the conifold field theory described in 41], so all of the adjoint fields are massive when we consider these representations.

Let us write these representations for generic $q$. The idea is that the algebra of the F-terms will be a slightly modified $S U_{q}(2)$ algebra, so the solutions will look essentially like representations of $S U(2)$. We can choose these to be of dimension $k$, where $Y, Z$ are like ladder operators.

$$
Y=\left(\begin{array}{cccc}
0 & y_{1} & 0 & \ldots \\
0 & 0 & y_{2} & \ldots \\
\vdots & \vdots & \ddots & \ddots
\end{array}\right) \quad Z=\left(\begin{array}{cccc}
0 & 0 & 0 & \ldots \\
\zeta_{1} & 0 & 0 & \ldots \\
0 & \zeta_{2} & 0 & \ldots \\
\vdots & \vdots & \ddots & \ddots
\end{array}\right)
$$

The commutation relations with $X$ force $X$ to have the following form

$$
X=x \operatorname{diag}\left(1, q, q^{2}, \ldots, q^{k-1}\right)
$$


Finally, the commutation relations of $Y, Z$ give linear relations that overdetermine the $y_{i} \zeta_{i}$. It is even possible to choose a gauge where $y_{i}=1$, so that only the $\zeta_{i}$ are variables. Consistency of the equations gives a finite number of roots $x$ that solve the problem, and the number of different roots is equal to the degree of $V^{\prime}(x)$. Irreducibility requires that all of the $\zeta_{i}$ are different from zero. For $q^{n}=1$, if $k \geq n$ one can show that the above representations are reducible, because the $\zeta_{i}$ end up being periodic and a zero invariably shows up.

The ones that survive for $q^{n}=1$ are exactly the ones that are associated to fractional branes in the geometry, and have rank less than $n$. If we evaluate $t$ as defined in the bulk for the fractional brane representations, we find that $t=f\left(q^{k-1} x\right)$; and the condition on $x$ to be a "root" are that $f(x)=f\left(q^{k} x\right)$.

Notice that there is no gauge symmetry sending $x \rightarrow q x$ for the fractional branes. This means that a fractional brane contributes to traces of the form $\operatorname{tr}\left(X^{k}\right)$ for all $k$. These gauge invariant variables are not traces of elements of the center of the algebra.

Also, at roots of unity for $q$, of the infinite number of representations of the slightly deformed $S U_{q}(2)$ we have for general $q$, only finitely many survive, and these are exactly the representations of dimension less than $n$. This is the property that will give a finite number of cuts later in the matrix model.

The fractional branes can lead to isolated vacua of a $U(N)$ theory for arbitrary $N$. These are exactly like the representations of $S U(2)$ that are familiar from the $\mathcal{N}=1^{*}$ system. There are also fractional branes at the curve of singularities. These have a moduli space of vacua which is given by a connected $n$-fold cover of the curve. Given $n$ branes in the curve of singularities one can move them so that they sit on top of each other in the CY geometry, but that each of them is in a different leaf of the cover. This configuration is produced by taking a brane in the bulk to the line of singularities.

The description of the classical vacua of the theory for $U(N)$ is given by splitting into irreducible representations of the algebra.

$$
R=\oplus N_{i} R_{i}
$$

and lead to a theory which in the infrared has a $\prod U\left(N_{i}\right)$ gauge symmetry, with $N=$ $\sum N_{i} \operatorname{dim}\left(R_{i}\right)$. If the $R_{i}$ are branes in the bulk or in the curve of singularities, then one has a moduli space of vacua. Even if the $R_{i}$ are fractional branes, $R_{\alpha}^{ \pm}$, there can be a moduli space for these branes if they are accompanied by $R_{\alpha}^{m p}$.

The branes in the bulk behave in the low energy physics like $\mathcal{N}=4$ field theory, so we can take them to a generic point in moduli space where they are away from the singularities, and also remove paired fractional branes to a generic point in moduli space in the bulk. After this is done, there are some fractional branes that can not be removed from the singularities by moving inside the classical moduli space. The number of branes stuck at each isolated singularity is constant in the moduli space (this is a classical statement).

In this form we realize that the description of the moduli space of the $U(N)$ theory comes in branches of different dimension, and the branches are characterized by how we choose to distribute fractional branes at the isolated singularities. 
Among all of these possibilities, one can always find solutions where there are only fractional branes stuck at the isolated singularities. This is because there is at least one fractional brane with $\operatorname{dim}\left(R_{\alpha}\right)=1$. In general we can have various isolated vacua where the fractional branes are distributed in some form at the singularities. These isolated vacua are exactly of the type that one believes one understands, because in the infrared one has pure gauge theory degrees of freedom. The $S U\left(N_{i}\right)$ gauge groups should confine and the low energy theory reduce to $\prod U(1)$, just like in the $\mathcal{N}=2 \mathrm{SYM}$ softly broken to $\mathcal{N}=1$.

Geometrically, if we place fractional branes at conifold singularities, these are expected to take the geometry through a geometric transition and deform the singularity away, if they have the same behavior as described in [40, 33]. This produces a new geometry where the quantum effects of the branes are taken into account. We will argue later, based on holomorphy that this should correspond to having a quantum corrected curve $\tilde{Q}(t, u)$, with a more generic polynomial of degree $n$ in $t$ and $s$ in $u$.

We can now ask the question: how will we be able to see this geometry? The intuition is that if we use a probe brane, then the brane will have as it's moduli space the deformed geometry. This intuition is valid so long as we can ignore the back-reaction of the branes at the singularities to the probe. From the string theory point of view, this will be a large $N_{i}$ limit. However, we will see that we can predict using matrix models that there is no back reaction even at finite $N_{i}$.

The issue we should now consider, is that for every distribution of fractional branes we get a different deformation. In the string geometry this is because the geometric transition exchanges the algebraic cycle that the fractional branes are wrapping, to an $S^{3}$ with flux. The flux is given by the number of branes wrapping the cycle, and the flux tries to make the volume of the associated $S^{3}$ bigger in order to reduce the energy in the flux. Thus, we have to consider each vacuum configuration independently.

In particular, the description of the quantum deformed moduli space of a probe brane has to be different for each branch of vacua. This is very similar to the discussion given in [8], where the order parameters that distinguish the vacua are equations which are only satisfied on some branches of the theory and not others. If we stop to think for a minute, the discussion we have done so far even at the classical level has this property. The equation of the CY geometry in the gauge theory assumes that we have split the classical representation of the vacua into irreducible representations, and the equation is only valid representation by representation. This is, we have made a choice of eigenvalues (we can think of block diagonal matrices as a generized form of eigenvalue), and the equations are valid eigenvalue by eigenvalue.

However, the eigenvalues themselves are not gauge invariant, as we have to account for permutations of the eigenvalues. Only the symmetric functions of the eigenvalues are truly gauge invariant. However, the CY equations described above are not valid when we sum over eigenvalues. They are only valid eigenvalue by eigenvalue. To classically obtain the eigenvalues from the symmetric functions amounts to solving a polynomial and choosing a labeling for the roots. This extra label is the non-gauge invariant information that tells us that classically we are not studying universal equations on the chiral ring of the gauge theory, but rather equations in the chiral ring that are valid only on a particular branch of 
the theory.

This subtle point is very important. It means that the non-universal equations that determine the quantum moduli space does not only receive contributions from instantons, but it can be corrected by other strong dynamics effects, such as gaugino condensation.

\subsection{The quantum moduli space}

We have exhausted the discussion of the classical moduli space of the theory. Now we want to study the moduli space of one probe in the presence of fractional branes at the singularities, whereas in the previous section we discussed essentially the moduli space of one probe alone, plus the classical description of the branches of the gauge theory. We want to understand the quantum effects of the fractional branes on the geometry, and we will use a probe brane to test the shape of the geometry.

Before we study these quantum deformations, one should make a field theory argument for the moduli space not being lifted, namely, for the absence of a potential on the moduli space.

The argument one can make is semiclassical. If one examines the theory at infinity in moduli space, all the massive fields that connect the brane in the bulk to the fractional branes have a mass much higher than any confining scale. This means one should be able to integrate them out completely and reduce the problem to low energy effective field theory. The effective field theory on the probe brane is essentially $\mathcal{N}=4$ SUSY gauge theory locally, and we do not expect that to change too much. The effective superpotential of the brane in the bulk should decay at infinity, because we are in a semiclassical regime. Now, the vev of the superpotential is controlled by holomorphy, and it vanishes at infinity. If the moduli space is lifted, then the superpotential must be a holomorphic function on the moduli space, and it should have poles or vanish exactly. The only possibility of having poles is if there are codimension one singularities on the classical moduli space. However, the moduli space described above is a Calabi-Yau geometry, which cannot have singularities in codimension one. This means that there is no effective potential generated on the moduli space, and the moduli space is not lifted. This does not prevent the moduli space from getting corrected. Indeed, our argument in the previous subsection suggests that it does, and when it does, the constraints that the deformed moduli space satisfies are controlled by holomorphy. This argument should be generic for moduli spaces of bulk-branes in a geometrically engineered theory, as in that case the moduli space of vacua of a brane in the bulk is always a CY geometry.

To check the possible shape of the geometry we need to consider the $U(1)_{R^{\prime}}$ charge we defined. It is clear that when we keep the charges of the coefficients of $V$ into account, the equation describing the moduli space is homogeneous for the $U(1)_{R^{\prime}}$ charge. Since this is a non-anomalous symmetry of the gauge theory, this remains true in the quantum moduli space. A (partial) gaugino condensate $S_{i}$ will have the same charge as the variable $t$ (this is the charge of the superpotential term).

However, $u$ can have a charge that is not commensurate with $S$, because we had some freedom in the definition of the $U(1)_{R^{\prime}}$ charge. Also $v, w$ are charged with respect to the $U(1)_{Y Z}$ symmetry, so they must appear together. 
Holomorphy implies that all of the couplings, and $S$ appear with positive powers in the deformed equation. Also, the moduli space is not expected to develop new branches, so it must also be polynomial in $u, v, w, t$, and it should reduce to the classical moduli space if we set the $S_{i}$ to zero. The $R^{\prime}$ charge of the $S$ allow us to replace monomials where a $t$ appears with an $S$; the coefficients depend on the branch of moduli space one is studying, but we can not change the coefficient of $v w$ nor include higher powers of $v w$. This results in a deformed CY geometry where the equation that describes the geometry has the form

$$
u v w=Q(t, u)+\delta(t, u)=\tilde{Q}(t, u)
$$

where we have deformed the curve $Q(t, u)$ to $\tilde{Q}(t, u)$, making sure that the $R^{\prime}$ charge is conserved when we keep the information of the charge of the gaugino condensate.

Now, let us take an example to illustrate the shape of the deformations, where we set $n=2$ and $s=3$. In this case $V(x)=a_{1} x+a_{3} / 3 x^{3}, 2 f(x)=a_{1} x+a_{3} x^{3}=-2 f(-x)=$ $x\left(a_{1}+a_{3} u\right)$, and the curve is given by

$$
Q(t, u)=\left(t-x / 2\left(a_{1}+a_{3} u\right)\right)\left(t+x / 2\left(a_{1}+a_{3} u\right)\right)=t^{2}-u\left(a_{1}+a_{3} u\right)^{2} / 4
$$

The singularity of $Q(t, u)$ occurs at $u=-a_{1} / a_{3}, t=0$. In principle the allowed deformation of the polynomial are given by $\delta(t, u)=r_{0} t u+r_{1} t+\beta_{1} u+\beta_{0}$. The other coefficients, which are of higher degree, are determined uniquely by $a_{1}, a_{3}$, the bare parameters of the superpotential. $r_{0}$ and $r_{1}$ can be eliminated by a linear change in variables in $t$, so we drop them. In general we can always eliminate any term proportional to $t^{n-1}$, so $\tilde{Q}(t, u)=t^{n}+t^{n-2} O_{1}(u)+t^{n-3} O_{2}(u)+\ldots$

Moreover, we have not placed branes at the curve of singularities located at $u=0$, so we are not allowed to deform these singularities away. This forbids us from adding the term $\beta_{0}$. In the end the only allowed deformation is given just by $\beta_{1} u$. This is a one parameter deformation of the geometry. This is expected because we have only one conifold singularity to resolve. The counting of parameters that deform the geometry should be equal to the maximum number of conifold singularities that the geometry can have. When these conifolds collide they can generate more complicated singularities, but the counting of normalizable deformations should remain invariant.

In the general case the polynomial $Q(t, u)$ has no term added which does not contain a power of $u$, because we do not place fractional branes at the curve of $A_{n-1}$ singularities, only at isolated singularities. This gives rise to constraints on the coefficients of the curve. The reader can verify that the deformed CY geometry still has a curve of singularities at $t=u=0$ with the above prescription of not including any deformation with just a different power of $t$ which does not involve $u$ as well. The general idea is that placing fractional branes at a given singularity only resolves that singularity, and leaves the other singularities intact. In fact this argument has been used in various papers [9, 8] to study factorization properties of Seiberg-Witten curves. The geometrical idea is that one stabilizes the 3-cycles by flux, and in the absence of flux, the cycle shrinks to zero size. At least from this point of view one can characterize the configurations with no branes at some singularities: these are solutions where the curve $\tilde{Q}(t, u)$ preserves these singularities, and is therefore singular. 
We have presented a holomorphy argument to determine the deformed geometry as seen by a probe in the bulk, when we have placed fractional branes at the conifold singularities. The reader should be warned that the arguments given above assume that there is a way to define the variables $u, v, w, t$ for a given configuration. Even classically, this depends on "removing" the information of the fractional branes to extract these variables. In the quantum theory this procedure gets corrections, so it becomes very tricky to define these in a sensible way. Even the partial gaugino condensates need a good definition in the field theory [7]. The underlying assumption of these ideas is that all of this can be done in a systematic way, and that there is a procedure to determine these variables in terms of vevs of elements of the chiral ring of the gauge theory. This has to be done in each particular branch of the theory separately, and with only one probe brane in the bulk.

We will later show that as in the example of the previous section, the deformed geometry encodes relations for some generalized resolvents in a matrix model. This result can be used to find recursion relations which 'solve' the matrix model in the sense expressed in the introduction.

\subsection{The chiral ring of the gauge field theory}

We have argued what the shape of the deformed moduli space of vacua is, as it pertains to a single bulk D-brane. In some sense, this solves the structure of the chiral ring for a single brane in the bulk.

As we have argued, the description of the classical moduli space depends on singling out eigenvalues and performing various operations with classical matrices. In the quantum theory all of these statements should be interpreted as operator equations in the chiral ring, so it is important to classify the chiral ring of the full gauge theory, not just of the "bulk" brane.

The description is very similar to the one matrix model, except that now we have three matrices. Also, we should regard the rank of the matrices as an unknown, as we can have arbitrary fractional branes at the various singularities.

The chiral ring is made of gauge invariant operators, and factorization is guaranteed by the cluster decomposition principle when we study chiral rings vevs in a supersymmetric vacuum. It is under these conditions that we actually believe that it is possible to relate the supersymmetric gauge theory to the associated matrix model.

Following the setup of [7], the chiral ring is generated by traces of the following form

$$
\begin{array}{r}
\operatorname{tr}(f(X, Y, Z)) \\
\operatorname{tr}\left(f(X, Y, Z) W_{\alpha}\right) \\
\operatorname{tr}\left(f(X, Y, Z) W_{\alpha} W^{\alpha}\right)
\end{array}
$$

where $f$ is a polynomial in $X, Y, Z$. We have to remember that $X, Y, Z$ are matrices, so the ordering inside $f$ of the different letters $X, Y, Z$ matters. The ordering of $W_{\alpha}$ is not important, because adjoint action of $W_{\alpha}$ on a set of letters is a total derivative. This allows us to choose an ordering where all the $W$ 's are together. 
Also, no more than two $W_{\alpha}$ per trace are allowed because these terms are a total chiral $\bar{D}$ derivative [7].

The chiral ring generators as presented above are independent of $N$, and of the specific details in the superpotential. The generalized Konishi anomaly equations for variations of the fields will depend on the specific form of the superpotential, and these are the ones that can be related to a matrix model.

Classically, the relations in the chiral ring are given by solving the F-term constraints of the superpotential. These can be written in a gauge invariant way in the following form

$$
\operatorname{tr}\left(\frac{\partial W}{\partial \phi} f(X, Y, Z)\right)=0
$$

for $f$ any word in the fields, and $\phi$ any of $X, Y, Z$. These are essentially the same equations as $W^{\prime}=0$, since it implies that no matter what matrix we build out of $X, Y, Z$, the trace is zero.

If we include quantum corrections, the equations of motion take the form

$$
\operatorname{tr}\left(W^{\prime} f\right)=\text { Quantum corrections }
$$

The quantum corrections involve the (partial) gaugino condensate (s) of the (fractional branes) gauge theory, and we should interpret the above equation as a part of a generalized Konishi anomaly, where $f$ can include the $W_{\alpha} W^{\alpha}$.

A few points are worth noticing: when we fix the superpotential as in the previous subsection, branes in the bulk do not contribute classically to one point functions of the form

$$
\operatorname{tr}\left(X^{k}\right), \operatorname{tr}\left(Y^{k}\right), \operatorname{tr}\left(Z^{k}\right)
$$

for $k \neq 0 \bmod (n)$, nor to any word containing a gaugino field ${ }^{5}$. This can be seen because the classical solutions of branes in the bulk give contributions to the above as sums of the form

$$
\sum_{i} q^{i k}=0
$$

In the orbifold theory, all of these words correspond to twisted fields. The classical string theory statement is that a brane in the bulk does not carry charge in the twisted sector of the orbifold, so it can not generate vevs for twisted fields.

Fractional branes, on the other hand, do couple to the twisted sector. It follows that they generate vevs for twisted sector words. We should read this statement in the following way: classically, there is information that depends only on the fractional brane structure.

Moreover, we are interested in isolating the fractional branes that are stuck to the codimension three singularity, in a situation where there is no moduli space of vacua. In the gauge theory the low energy physics reduces to pure gauge theory $\prod U\left(N_{i}\right)$, and we can argue that the $S U\left(N_{i}\right)$ confine and give rise to some partial gaugino condensate.

\footnotetext{
${ }^{5}$ Classically the gaugino field has to be zero for Lorentz invariance, and this implies that the gaugino condensate classically is also zero
} 
Guaranteeing that there are no branes in the codimension two singularities of the geometry is a simple matter. These contribute to the vevs $\operatorname{tr}\left(Y^{k}\right)$ and $\operatorname{tr}\left(Z^{k}\right)$ for all $k$. Also, branes in the bulk can contribute to $\operatorname{tr}\left(Y^{k}\right), \operatorname{tr}\left(Z^{k}\right), \operatorname{tr}\left(X^{k}\right)$ for $k$ multiples of $n$.

This means that classically these can be considered variables that we can set to any value we want. Notice that $\operatorname{tr}\left(X^{n}\right)$ and $\operatorname{tr}\left(X^{2 n}\right)$ are uncorrelated if we have more than one brane in the bulk. In particular, we can always consider enough branes in the bulk so that these variables are allowed to have any value.

The same is not true for the other powers of $\operatorname{tr}\left(X^{k}\right)$. There are only a finite number of values of $X$ that are allowed for a fractional brane, so these variables are related to each other in the classical theory; there will be recursion relations that determine these variables. The data necessary to seed this information to the classical recursion relations is exactly how many fractional branes there are at each singularity, and which cycle they are wrapping.

One can wonder if in the quantum theory the vevs of $\operatorname{tr}\left(X^{k}\right)$ and $\operatorname{tr}\left(X^{k} W_{\alpha} W^{\alpha}\right)$ for $k$ not a multiple of $n$ can get contributions from branes in the bulk. We will argue that for the second ones, which have a simple matrix model interpretation this is not so. A hand waving argument would say that the gauge groups that have gaugino condensation are just the fractional branes and not the branes in the bulk, so they contribute to these one point functions. In principle, if these were classical matrices, one could make the same statement about any trace containing $W^{\alpha} W_{\alpha}$. However, the quantum vacuum is not given by classical matrices, and one should consider only twisted operators.

At infinity the branes in the bulk should be semiclassical and decouple, so their effective gaugino condensate should vanish. Thus the contribution to $\operatorname{tr}\left(X^{k} W^{2}\right)$ should be suppressed by a large mass scale. At infinity, their contribution is zero, and $\operatorname{tr}\left(X^{k} W^{2}\right)$, being holomorphic on the moduli space, is constant by the same argument that made the effective superpotential on the moduli space equal to zero. This suggests that these one point functions are determined by the fractional branes alone.

Since these are related to $\operatorname{tr}\left(X^{k}\right)$, and classically the twisted $\operatorname{tr}\left(X^{k}\right)$ satisfy recursion relations (they are determined alone by the fractional branes), it suggests that the same is true for $\operatorname{tr}\left(X^{k} W^{2}\right)$. The description above is suggestive. It is not meant to be a formal argument. We will use matrix models to make this intuition precise.

\subsection{Relations to a matrix model}

We have found that we can write the most general form of the deformation of the CalabiYau geometry which is compatible with holomorphy. This information in the gauge theory encodes non-perturbative dynamics that results from gaugino condensation on the gauge group of the fractional branes. All of these effects should be computable from a matrix model. What we have described is the moduli space of a probe brane. The matrix model calculation proceeds in the same way we worked in section 2. We isolate the block of matrices associated to the probe brane. This is done by (perturbatively) identifying a set of eigenvalues for $X$ ( an $n \times n$ block matrix) to correspond to a bulk brane, where the eigenvalues are related to each other in such a way that they produce massless field in the classical field theory. 
In the end, we have to consider the three matrix model whose potential is given by

$$
W(X, Y, Z)=N \mu^{-1}[\operatorname{tr}(X Y Z-q X Z Y)+\operatorname{tr} V(X)]
$$

with $n$ correlated eigenvalues which correspond to a single brane in the bulk, and take the large $N$ limit.

We are after the effective potential for the set of block matrices in the bulk. Notice that we can choose the gauge where $X$ is diagonal, and we can isolate the block of matrices which correspond to the eigenvalues in the bulk.

The idea now is to integrate the off-diagonal elements that connect the eigenvalues of the bulk to the eigenvalues of the fractional branes. The procedure is to integrate these fields in the matrix model, where we take a large $N$ limit for the matrices and we will obtain an effective potential for $X_{b u l k}, Y_{b u l k}, Z_{b u l k}$ with the effects of the fractional branes included.

This should be seen in the same philosophy of the proof of the matrix model conjecture as given in [15]. The new ingredient is that perturbatively massless fields can not be integrated out. We also have to remember that since the classical moduli space has singularities, at each singularity new massless fields appear. The $n \times n$ block of matrices contains all of these fields, so we need to keep them unintegrated to understand what happened to the singularities.

To compute the quantum corrected moduli space then we need to vary the effective potential for $X_{b u l k}, Y_{b u l k}, Z_{b u l k}$ as $n \times n$ matrices and set the variations equal to zero. We then need to evaluate what are the quantum corrections to the form of $u, v, w, t$ for the bulk matrices and find the new relations between them. This is technically a little bit more elaborate than the situation in section 2. It is useful to keep in mind that the brane in the bulk is auxiliary: it is giving us information about the matrix model of the branes stuck at the singularities. We want to know exactly what information it is encoding.

To do the first part, we divide the matrices as follows

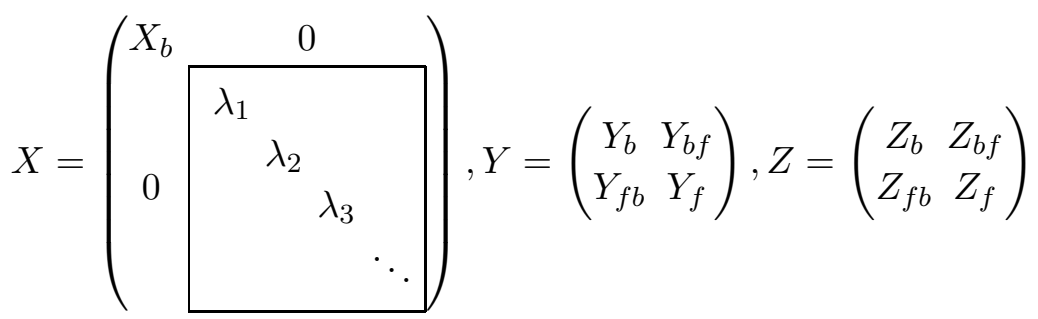

where we have taken care to write the matrix $X$ in a block diagonal form, and where the eigenvalues attached to the singularities are singled out. We keep the matrix of the bulk $X_{b}$ in general form. In the matrix model, with $X_{b}$ unintegrated, requiring that the $X_{b f}=0$ is a gauge choice. This introduces ghosts [16], whose measure will give the Vandermonde determinant. The subindices are labeled as a mnemonic: $b$ for bulk, $f$ for fractional. Integrating over the orbit of the gauge group introduces a factor of the volume of the orbit, which is proportional to the Vandermode determinant. This includes both the eigenvalues at the singularities and the block matrix $X_{b}$, even if we choose not to diagonalize $X_{b}$, there is still a contribution from how the eigenvalues $\lambda$ 'repel' the block $X_{b}$. 
When we take the logarithm of this measure term, it gives us a contribution to the effective potential which is given by

$$
2 \sum_{i} \operatorname{tr}_{b} \log \left(X_{b}-\lambda_{i}\right)+\sum_{i \neq j} \log \left(\lambda_{i}-\lambda_{j}\right)
$$

We have only written the terms involving $X_{b}$, as this is the information that relates to the brane in the bulk ${ }^{6}$.

We now set $X_{b f}=0$ everywhere in $W(X, Y, Z)$ and remember to keep track of the Vandermonde determinant.

It is easy to see that after this substitution $Y_{b f}, Y_{f b}$ and $Z_{f b}, Z_{b f}$ appear quadratically in the equation, and can be integrated completely, separating the matrices from the bulk from the matrices associated to the fractional branes, except for quantum effects. These two off-diagonal integrations contribute another effective term to the potential of $X_{b}$ alone, which is given by

$$
-\sum_{i} \operatorname{tr}_{b}\left(\log \left(X_{b}-q \lambda_{i}\right)-\sum_{i} \log \left(X_{b}-q^{-1} \lambda_{i}\right)\right)
$$

Again, we obtain a formal power series in $X_{b}$ which encodes loop operators of the fractional brane matrices.

The effective potential for $X_{b}, Y_{b}, Z_{b}$ in the matrix model is then given by

$$
\begin{aligned}
W=N \mu^{-1} & \left(\operatorname{tr}\left(X_{b} Y_{b} Z_{b}-q X_{b} Z_{b} Y_{b}\right)+V\left(X_{b}\right)\right) \\
& -\sum_{i}\left[2 \log \left(X_{b}-\lambda_{i}\right)-\log \left(X_{b}-q \lambda_{i}\right)-\sum_{i} \log \left(X_{b}-q^{-1} \lambda_{i}\right)\right]
\end{aligned}
$$

Now, we have to assume that the distribution of the $\lambda_{i}$ is known, so that we can think of the terms involving logs as a formal function of $X_{b}$. Notice that this formal function has a power series expansion in $X_{b}$ which has the same characteristics as $V\left(X_{b}\right)$ : no powers of $X_{b}$ which are multiples of $n$ appear. The effect is the same as if we would have done a change in the potential

$$
V \rightarrow \tilde{V}=V-\frac{\mu}{N} \sum_{i}\left[2 \log \left(X-\lambda_{i}\right)-\log \left(X-q \lambda_{i}\right)-\sum_{i} \log \left(X-q^{-1} \lambda_{i}\right)\right]
$$

so the moduli space has essentially the same form as we discussed in the previous section, except that the new $\tilde{Q}$ now has information about the distribution of eigenvalues $\lambda_{i}$. Notice that the formal form of the solution gives us a curve of the following form

$$
u v w=Q_{\text {matrix }}(t, u)
$$

where in principle $Q_{\text {matrix }}(t, u)$ is a formal power series in $u^{-1}$.

\footnotetext{
${ }^{6}$ The saddle point equations for the eigenvalues $\lambda$ are discussed in the appendix.
} 
The new calculation of $\tilde{V}(X)^{\prime}$ gives the following result

$$
\begin{aligned}
\tilde{V}^{\prime}(X) & =V^{\prime}(X)+\frac{\mu}{N}\left[\sum_{i} 2 \frac{1}{X-\lambda_{i}}-\frac{1}{X-q \lambda_{i}}-\frac{1}{X-q^{-1} \lambda_{i}}\right] \\
& =V^{\prime}(X)+\frac{\mu}{N} \sum_{i} \sum_{j=1}^{n-1}\left(2-q^{j}-q^{-j}\right) \frac{X^{n-j-1} \lambda_{i}^{j}}{x^{n}-\lambda_{i}^{n}}
\end{aligned}
$$

where $u=x^{n}=X^{n}$ is still in the center of the modified algebra.

We can define the following $n-1$ generating functions of moments of the eigenvalues $\lambda_{i}$

$$
R_{j}(\gamma)=\frac{1}{N} \sum_{i} \frac{\lambda_{i}^{j}}{\gamma-\lambda_{i}^{n}}=\sum_{k=0}^{\infty} \frac{<\lambda^{n k+j}>}{\gamma^{k+1}}
$$

and write the (matrix version of the) quantum moduli space in terms of these generating functions.

The quantum version of $f(x)$ is

$$
\tilde{f}(X)=f(X)+\sum_{j=1}^{n-1}\left(1-q^{j}\right) X^{n-j} R_{j}(u)
$$

Now we can compare both descriptions of the quantum moduli space: the one we obtained using holomorphy arguments, and the one where we assumed that we had the knowledge of the $R_{j}(u)$. The main assumption in this paper is that both constructions are giving exactly the same moduli space. Indeed, the one difference between $\tilde{Q}(t, u)$ and $\tilde{Q}_{\text {matrix }}(t, u)$ is that the first one is a polynomial, and the second one is only a formal power series.

The equality of the moduli spaces means that we can write equations that the $R_{j}(u)$ must satisfy: one for each power of $t$, so that the matrix model gives also a polynomial. There are $n-1$ unknown functions $R_{j}(u)$, and there are $n-1$ non-trivial relations, one for each power of $t$ starting at $t^{n-2}$. The equation for $t^{n-1}$ is satisfied trivially because the product defining $Q$ is over all the values of $\tilde{f}\left(q^{i} X\right)$, and $\tilde{f}$ does not contain integral powers of $X^{n}$. This means the solution is algebraic.

The other equations are given by expanding both forms of $\tilde{Q}(t, u)$ and comparing coefficients. We will call these the Quantum relations of the matrix model. The first two are explicitly written bellow:

$$
\begin{aligned}
O_{1}(u) & =\sum_{i<j} \tilde{f}\left(q^{i} x\right) \tilde{f}\left(q^{j} x\right) \\
-O_{2}(u) & =\sum_{i<j<k} \tilde{f}\left(q^{i} x\right) \tilde{f}\left(q^{j} x\right) \tilde{f}\left(q^{k} x\right)
\end{aligned}
$$

The right hand side is given by explicit power series of $u^{-1}$ in terms of the one point functions $\left\langle\lambda^{k}>\right.$, and all of the coefficients except the first few (the ones that have positive powers of $u$ ) vanish. This gives us constraints that the one point functions must 
satisfy, and one can notice that they give rise to recursion relations for the moments of the eigenvalues $\left\langle\lambda^{k}>\right.$, so that all but a finite number of them are determined in terms of initial conditions. The polynomials $O_{i}$ serve to encode these initial conditions.

In more detail, we can decompose $f$ in terms of powers of $x$ modulo $n$,

$$
\begin{aligned}
& f_{j}(x)=\left(1-q^{j}\right)^{-1} \sum_{i \equiv j \bmod (n)} a_{i} x^{i} \\
O_{1}(u)= & \sum_{i<j} \tilde{f}\left(q^{i} x\right) \tilde{f}\left(q^{j} x\right) \\
= & \sum_{j} \frac{1}{2}\left[\left(f_{j}\left(1-q^{j}\right)^{-1}+\mu\left(1-q^{-j}\right) R_{n-j}(u) x^{j}\right)\right. \\
& \left(f_{n-j}\left(1-q^{-j}\right)^{-1}+\left(1-q^{j}\right) \mu R_{j}(u) x^{n-j}\right) \\
& \left.\left(q^{j}+q^{2 j}+\cdots+q^{(n-1) j}\right)(n-1)\right]
\end{aligned}
$$

The sum appearing in the above equation can be evaluated using $1+q^{j}+q^{2 j}+\cdots+q^{(n-1) j}=$ 0 . We get

$$
\begin{array}{r}
O_{1}(u)+(n-1) \frac{1}{2}\left(2-q^{j}-q^{-j}\right)^{-1}\left(f_{j}(x) f_{n-j}(x)\right)= \\
\mu(n-1)\left[\sum_{j} f_{j}(x) x^{n-j} R_{j}(u)-\frac{\mu u R_{j}(u) R_{n-j}(u)\left(2-q^{j}-q^{-j}\right)}{2}\right]
\end{array}
$$

Now, the left hand side of the above equation is a polynomial in $u$, while the right hand side is a power series in $u^{-1}$. Equality means that the coefficients of $u^{-k}$ of the right hand side must vanish identically.

After a little bit of algebra collecting the powers of $u^{-1}$ this gives us the following relations

$$
\sum_{i} a_{i}<\lambda^{i+n s}>=\frac{\sum_{j}\left(2-q^{j}-q^{-j}\right)<\lambda^{n s-j}><\lambda^{j}>}{2}
$$

Now, we will show that these equations can be derived from manipulating loop equations in the associated matrix model. We can ask why these relations look so different from each other for different values of $n$, and what is special about $q$ being a root of unity in the matrix model. This is what we will analyze in the next section. Notice that the concept of holomorphy has allowed us to propose some polynomial relations for twisted one point functions in the matrix model, but at this moment this should be interpreted as a guess, since there is no proof that the construction of the moduli space based on matrix models necessarily gives the right answer.

\section{Obtaining the Quantum relations from loop equations for a matrix model}

Consider the three matrix model whose potential is given by

$$
W(X, Y, Z)=N \mu^{-1}[\operatorname{tr}(X Y Z-q X Z Y)+\operatorname{tr} V(X)]
$$


with

$$
V(x)=\sum_{i \neq 0} \frac{a_{i}}{i} x^{i}
$$

a polynomial, and $q^{n}=1$ a primitive $n$-th root of unity.

Because the matrix model is given formally by the superpotential of a supersymmetric field theory, we will classify the one point functions $\langle f(\phi)\rangle$ by their quantum numbers under the $\mathbb{Z}_{n} \times \mathbb{Z}_{n}$ quantum symmetry of the corresponding chiral operator in the quantum field theory. A word which has charge zero under the quantum symmetry will be called untwisted, and a word which transforms non-trivially under the quantum symmetry will be called twisted. This nomenclature is borrowed from the fact that the theory with $V(x)=0$ results from the field theory at a special orbifold singularity. We will also assign $Y$ and $Z$ charges \pm 1 , so that we will also use this $U(1)_{Y Z}$ charge to classify words.

Since this second charge is conserved by the potential and the measure, we can consistently set to zero the one point function of any word whose charge is different from zero. This constraint at the level of the classical field theory implies that there are no fractional branes on the singular curves of the CY, and is a simplifying assumption to reduce the amount of algebra. We want to impose this constraint as well on the matrix model, since we are interested in situations where the matrix model has an isolated vacuum about which all of the modes are massive.

We will use throughout the following simplifying notation convention for the one point functions of words in the matrix model

$$
<f(\phi)>=<\frac{1}{N} \operatorname{tr}(f(\phi))>
$$

Now we will proceed to derive the equations 3.37 from the loop equations associated to this matrix model.

The universal loop equations can be written by considering an infinitesimal change of variables $\delta \phi^{i}=f(\phi)$ (one assumes this is a monomial written as some ordering of the matrix variables, i. e. a word in the matrices), and realizing that the following expression is the integral of a total derivative and therefore vanishes

$$
\int[d \phi] \partial_{\left(\phi^{i}\right)}^{j}\left(\delta \phi^{i}\right)_{j}^{k} \exp (-W)=\int[d \phi] \operatorname{tr}\left(\partial_{\phi^{i}} \delta \phi^{i}\right) \exp (-W)=0
$$

The loop equations will have the universal form

$$
\sum \mu<f_{1}><f_{2}>=<\frac{\partial W}{\partial \phi^{i}} \delta \phi^{i}>
$$

where the sum is over all $f_{1}, f_{2}$ monomials such that $f_{1} \phi^{i} f_{2}=f(\phi)$, this is, from splitting the word $f$ into two words connected by the letter $\phi^{i}$. The above result is using factorization of the large $N$ limit of the matrix model.

Because we set all words with charge different than zero to zero, we need to look only at variations of the matrices $X, Y, Z$ which have the same charge as the object we are varying. Thus we will look for $\delta X=f(X, Y, Z)$, where $f$ has the same number of $Z$ and $Y$ 
matrices. Similarly $\delta Y=g(X, Y, Z)$ where the word $g$ has one more field of type $Y$ than of type $Z$.

There is also the $U(1)_{R^{\prime}}$ to consider. It's existence gives a grading to the set of words. One can systematically explore the set of possible monomial variations by exploiting the $U(1)_{R^{\prime}}$ symmetry and take variations with increasing $U(1)_{R^{\prime}}$ charge.

\subsection{The first equation}

We will study the loop equations inspired in the solution of the two matrix problem given in [51]. The main idea is to write the loop equations in such a way that one can learn information on complicated words in terms of simpler words.

Consider the following matrix variations, of minimal number of $Y, Z$ pairs

$$
\delta Y=X^{m} Y X^{k-m}
$$

The loop equations associated to this variation take the simple form

$$
\mu<X^{m}><X^{k-m}>=<X^{m+1} Y X^{k-m} Z-q X^{m} Y X^{k-m+1} Z>
$$

On the right hand side there are $k+2$ independent possible orderings of the fields, while we only have $k+1$ different equations. These one point functions on the right have exactly one pair of $Y, Z$ matrices. Variations of $Z$ of this type will produce the same equations as above, so they do not lead to new loop equations.

Also, one can consider the variation of $X$ given by $\delta X=X^{k+1}$, from which we get

$$
\mu \sum_{m=0}^{k}<X^{m}><X^{k-m}>-<V^{\prime}(X) X^{k+1}>=<X^{k+1} Y Z>-q<X^{k+1} Z Y>
$$

which gives an extra equation for some of the same one point functions in equation 4.7.

In total we have $k+2$ equations for the $k+2$ variables $\left\langle X^{m} Y X^{k+1-m} Z\right\rangle$, so in a generic case we could say that the one point functions of $\left\langle X^{m} Y X^{k+1-m} Z\right\rangle$ are all determined from the one point functions of $\left\langle X^{m}\right\rangle$. Considering the $U(1)_{R^{\prime}}$ grading, the words involving $Y, Z$ have higher degree than those with only $X$.

Notice that one can use the equations to shift $Z$ to the right in the word $\left\langle X^{m} Y X^{k+1-m} Z>\right.$, and then eventually one comes back to the original configuration with a phase, so in this case one can solve for any of the one point functions in the right hand side, as long as the phase is not zero.

However, there are situations in which these equations are not linearly independent. This will happen when $k$ is a multiple of $n$, and then on transporting $Z$ around the loop the total accumulated phase is $q^{k}=1$. From here the equations for the vevs $<X^{m} Y X^{k+1-m} Z>$ are linearly dependent. This means that the one point function $<X^{m} Y X^{k+1-m} Z>$ is not determined in this case. Notice that this is exactly the case when the word is untwisted ${ }^{7}$.

\footnotetext{
${ }^{7}$ From the point of view of the classical SUSY gauge field theory, this function in the chiral ring receives contributions from the moduli space of a probe brane, so it can take any value.
} 
Consistency of the loop equations then imposes a constraint between the elements of the left hand side. This constraint is given by summing

$$
\begin{aligned}
\sum \mu q^{m}<X^{m}><X^{k-m}> & =\sum q^{-m}<X^{m+1} Y X^{k-m} Z-q X^{m} Y X^{k-m+1} Z> \\
& =q^{-k}<X^{k+1} Y Z>-q<Y X^{k+1} Z> \\
& =<X^{k+1} Y Z>-q<Y X^{k+1} Z> \\
& =\mu \sum_{m=0}^{k}<X^{m}><X^{k-m}>-<V^{\prime}(X) X^{k+1}>
\end{aligned}
$$

and this equation can be rewritten after some manipulations as follows

$$
<V^{\prime}(X) X^{k+1}>=\frac{1}{2} \sum \mu\left(2-q^{m}-q^{-m}\right)<X^{m}><X^{k-m}>
$$

which is exactly the first of the relations that appeared in 3.46 .

As the reader can verify, the equation above is written only in terms of twisted onepoint functions, as $\left(2-q^{m}-q^{-m}\right)=0$ whenever $m$ is a multiple of $n$. Since $V$ is a polynomial (lets say of degree $s$ ), the above equation can be seen as a recursion relation that determines the highest degree component of $V^{\prime}(X) X^{k+1}$, namely the one point function of $\left\langle X^{k+s}\right\rangle$ (in the case where $k=r n$ ), in terms of one point functions of lower degree. The first such component that can be determined is the one corresponding to $\left\langle x^{s}\right\rangle$, and then all other components modulo $n$.

Now, we will solve the constraints for the one point functions $\left\langle X^{k+1} Y Z\right\rangle$ for $k$ not a multiple of $n$, because we will need them for future calculations. This is easily seen to be given by solving 4.9 when $k$ is not a multiple of $n$. In this case there is no equality between the second and third lines, and instead we get two linearly independent equations with two unknowns. We get that

$$
\begin{aligned}
\left(q^{-k}-1\right)<X^{k+1} Y Z>= & \sum \mu q^{m}<X^{m}><X^{k-m}> \\
& -\mu \sum_{m=0}^{k}<X^{m}><X^{k-m}>+<V^{\prime}(X) X^{k+1}> \\
= & \mu \sum\left(q^{m}-1\right)<X^{m}><X^{k-m}>+<V^{\prime}(X) X^{k+1} \ngtr
\end{aligned}
$$

Notice that on the right hand side of the third line we do get untwisted one point functions, so these can in principle receive contributions from branes in the bulk. This happens when $k-m$ is a multiple of $n$, or from $V^{\prime}(X) X^{k+1}$ when certain terms in the potential are non-zero.

\subsection{The second Quantum equation}

The next level of difficulty is obtained by taking words of next to minimal numbers of pairs of $Y, Z$ fields. For example $\delta Y=Y X^{m} Z X^{n} Y$, and all possible orderings of such monomials. Although it looks much harder to solve this linear system, we do not need to study the most general variations of $Y$ of this type. The main effect of the loop equations is to tell us what happens when we permute two of the symbols in a word in terms of 
one point functions of lower numbers of pairs of $Y, Z$. Thus any one point function can be determined from any other permutation by a series of steps. We only need to worry about the possibility of the system of equations not being linearly independent, just as we saw previously. This can happen when the words we study are untwisted, and then one produces a constraint between words of smaller degree.

To check this redundancy, we just need to take an initial word $<X^{k+2} Y Y Z Z>$ and cycle the $Z$ around the $X$ and $Y$. We will return with the same phase to the initial configurations when $k$ is a multiple of $n$. This is because passing a $Z$ through an $X$ gives a factor of $q$, and passing a $Z$ past a $Y$ gives a factor of $q^{-1}$, so the total phase from cycling $Z$ around the trace is $q^{k+2} q^{-2}=q^{k}$.

We only need to look at the variations $\delta Y=X^{m} Y^{2} Z X^{k+1-m}$, and their corresponding loop equations

$$
\mu<X^{m}><Y Z X^{k-m}>=<X^{m+1} Y^{2} Z X^{k+1-m} Z>-q<X^{m} Y^{2} Z X^{k+2-m} Z>
$$

where we have set the charged one point functions to zero.

We also need two equations to cycle the $Z$ past the $Y$. These are provided by the variations $\delta X=Y Z X^{k+2}$ and $\delta X=Z X^{k+2} Y$. These two loop equations will look as follows 8

$$
\begin{array}{r}
<Y Z Y Z X^{k+2}>-q<Z Y Y Z X^{k+2}>= \\
\mu \sum_{m}<Y Z X^{m}><X^{k+1-m}>-<Y Z X^{k+2} V^{\prime}(X)> \\
<Y Y Z Z X^{k+2}>-q<Y Z Y Z X^{k+2}>= \\
-<Z X^{k+2} Y V^{\prime}(X)>
\end{array}
$$

Summing 4.14 we get, in the case where $k$ is a multiple of $n$ that

$$
\begin{gathered}
<X^{k+2} Y^{2} Z X^{k+1-m} Z>-q^{2}<Y^{2} Z X^{k+2} Z>= \\
\mu \sum_{m=0}^{k+1} q^{k+1-m}<X^{m}><Y Z X^{k-m}>
\end{gathered}
$$

which is also the sum of the equations in 4.15

$$
\begin{gathered}
<X^{k+2} Y^{2} Z X^{k+1-m} Z>-q^{2}<Y^{2} Z X^{k+2} Z>= \\
q \mu \sum_{m}<Y Z X^{k+1-m}><X^{m}> \\
-q<Y Z X^{k+2} V^{\prime}(X)>-<Z X^{k+2} Y V^{\prime}(X)>
\end{gathered}
$$

We can rewrite the constraint as follows

$$
\begin{aligned}
& q \mu \sum_{m}\left(1-q^{-m}\right)<X^{m}><Y Z X^{k+1-m}>= \\
& q<Y Z X^{k+2} V^{\prime}(X)>+<Z X^{k+2} Y V^{\prime}(X)>
\end{aligned}
$$

Again, we notice that the one point functions of $\left\langle X^{m}\right\rangle$ for $m$ a multiple of $n$ do not contribute, because $q^{m}=1$. This means the terms proportional to $\mu$ only depends on

\footnotetext{
${ }^{8}$ We write only the equations with $\left\langle X^{k} Z>=0\right.$ for all $k$
} 
twisted one point functions. Notice that the right hand side also only depends on twisted one point functions, at least as far as the powers of $X$ that are excluded from $V(X)$. Notice that the constraint above is not in the form which we want, which depends only on one point functions of the type $\left\langle X^{m}\right\rangle$. However, we can use the results from the previous subsection to express all of the one point functions appearing in the above equations in terms of one point functions of $\left\langle X^{m}\right\rangle$, possibly including the ones where $m$ is a multiple of $n$, which we have no way of determining from our previous arguments. The equations obtained in this manner will contain terms of order $\mu^{2}$ (triple trace), of order $\mu$ (double trace), and of order $\mu^{0}$ (single trace). We still have to check that after we do all of these substitutions, the one point functions appearing in the above equation are all twisted, and that the equation coincides with what we have shown already. This would lend support to the existence of a recursion relation determining only the twisted powers of $X$.

At this point we need to substitute explicit forms for $V^{\prime}(X)$ in terms of it's polynomial expansion, because the way we will substitute in equation 4.12 depends on what particular powers of $X$ are available. We also need to do an extra step in equation 4.18 and move the $Z$ past some of the $X$ in the one point function of $\left\langle Z X^{k+2} Y V^{\prime}(X)>\right.$.

Consider expanding $V^{\prime}(X)=\sum_{i \neq 0 \bmod (n)} a_{i} X^{i-1}$. It is clear that we will get one point functions of monomials of the type $\left\langle Y X^{i-1} Z X^{k+2}\right\rangle$, in the case where $k$ is a multiple of $n$. The following equality is easy to show for these one point functions by summing terms of the equations 4.7 .

$$
<Y Z X^{i+k+1}>-q^{i-1}<Y X^{i-1} Z X^{k+2}>=\mu \sum_{m=0}^{i-2} q^{m}<X^{m}><X^{k+i+1-m}>
$$

This can be used to rewrite

$$
<Y X^{i-1} Z X^{k+2}>=q^{1-i}\left[<Y Z X^{i+k+1}>-\mu \sum_{m=0}^{i-2} q^{m}<X^{m}><X^{k+i+1-m}>\right]
$$

From here we can check that the coefficient of $a_{i}<Y Z X^{k+1+i}>$ in equations 4.18 coming from the potential is given by $q+q^{i-1}$.

We will now only check that the terms of order $\mu^{0}$, which are untwisted and proportional to the second power of the coefficients in the potential $(a)^{2}$ are canceled. For this, we need to use equation 4.12 and only keep terms that involve two powers of the $a$.

Indeed, we get the sum

$$
\begin{aligned}
\left.\sum_{i}\left(q+q^{1-i}\right) a_{i}<Y Z X^{k+i+1}>\right) & =\sum_{i}\left(q+q^{1-i}\right)\left(q^{-(k+i)}-1\right)^{-1} a_{i}<X^{k+i+1} V^{\prime}(X)>+O(\mu) \\
& =\sum_{i, j}\left(q+q^{1-i}\right)\left(q^{k+i}-1\right) a_{i} a_{j}<X^{k+i+j}>+O(\mu)
\end{aligned}
$$

Now we want to consider the case where $i+j$ is a multiple of $n$, and we are summing two different terms with the coefficient $a_{i} a_{j}\left\langle X^{k+i+j}\right\rangle$. Also, remember that $k$ is a multiple of $n$, so we can drop it from the powers of $q$. The coefficient for the sum of these two terms is equal to

$$
q\left(1+q^{-i}\right)\left(q^{-i}-1\right)^{-1}+q\left(1+q^{-j}\right)\left(q^{-j}-1\right)^{-1}
$$


where we substitute $i=-j \bmod n$, so that the numerator of the expression is equal to

$$
q\left(1+q^{i}\right)\left(q^{-i}-1\right)+q\left(1+q^{-i}\right)\left(q^{i}-1\right)=q\left(q^{-i}-q^{i}+q^{i}-q^{-i}\right)=0
$$

This shows that there are no untwisted powers of $X$ appearing in the one point functions of this second constraint which are of second order in the $a_{i}$. As these are also the terms that contain the highest power of $X$ that is available. This shows that the above equations will determine higher twisted one-point functions of $X$.

We still have not shown that in the double trace and triple trace operators one can eliminate all the untwisted powers of $X$. Since we have already found relations using the matrix theory calculation of the moduli space, and moreover we have shown that the constraints give the right result for the first quantum equation plus a consistency condition for the second equation, we will leave this lengthy algebraic calculation as an exercise to the reader.

The methodology for determining the recursion relations should now be evident to the reader. One has to add more and more pairs of $Y, Z$ operators, and consider variations of the form

$$
\delta Y \sim X^{k+s-m} Y^{s+1} Z^{s} X^{m}
$$

which allow us to circulate one $Z$ past all the $X$. We also need to consider the variations of $X$ that allow us to flip $Z$ past the $Y$ in words with charge 0 . Again, when the words are untwisted, the words will be undetermined classically and the loop equations are linearly dependent. Consistency imposes constraints that will involve twisted words with smaller numbers of pairs of $Y, Z$. Since these words are twisted, they will belong to the set of relations that can be solved in terms of smaller numbers of pairs of $Y, Z$ variables. One should be able to show that after performing some amount of algebra one can reduce the system so that there are only one point functions for powers of $X$ which are untwisted. The calculations at each succeeding step become more involved ${ }^{9}$, but this is a recursive algorithm to get the constraints.

Summarizing, we see that the presence of a moduli space of vacua has given us a degenerate system of linear equations for the one point functions $<X^{K} Y^{M} Z^{M-1} X^{K^{\prime}} Z>$. For generic values of $q$ the associated linear equations determine these one point functions completely in terms of functions of lower degree. However, one does not get constraints that involve one point functions of $X$ alone. These should be considered as unknown parameters determined by the "infinite number of cuts" of the matrix model. For the special values of $q$ that we studied, this does not happen, and the linear system is degenerate. For this linear system to have a solution, the terms of lower degree must satisfy a constraint. It is exactly these constraints that encode the shape of quantum moduli space of vacua of the associated SUSY gauge theory. Moreover, the presence of only a finite number of singularities in moduli space guarantee that for certain generalized resolvents, one only has a finite number of cuts. Also, due to the special form of the potential, in the particular

\footnotetext{
${ }^{9}$ It would be interesting to find a more effective method to calculate these relations in the matrix model to give a more compelling proof, say in terms of eigenvalue distributions for $X$. The author has not found a better way to understand it however.
} 
case we are studying, the solution effectively reduces to a one matrix model whose solution is determined by a curve $Q_{\text {quatum }}(t, u)$.

\section{Partial gaugino condensates}

We have now described a partial solution of the three matrix problem, and we have found that some interesting generalized resolvents (the $R_{j}(u)$ ) can be obtained from the curve $\tilde{Q}(t, u)$.

In particular, it is interesting to ask how many eigenvalues in the matrix model are located around each classical saddle point of the theory. In the gauge theory this will be the calculation that determines the gaugino condensates from the geometry.

Now we want to relate the $R_{j}(u)$ to the problem of the distribution of eigenvalues of $X$. In particular, $R_{j}(u)$ only has information about the distribution of fractional branes, so it only contains information about the eigenvalues of $X$ associated to the fractional branes.

Let us look at the definition of $R_{n-1}(u)$. It is given by

$$
R_{j}(u)=\frac{1}{N} \sum_{i} \frac{\lambda_{i}^{j}}{u-\lambda_{i}^{n}}=\sum_{k=0}^{\infty} \frac{<\lambda^{n k+j}>}{u^{k+1}}
$$

Classically, this function has poles at the eigenvalues $\lambda_{i}^{n}$. It is more convenient to introduce a cover of the $u$ plane where $u=\gamma^{n}$, and then write

$$
R_{j}\left(\gamma^{n}\right)=\frac{1}{N} \sum_{i} \frac{\lambda_{i}^{j}}{\gamma^{n}-\lambda_{i}^{n}}
$$

In particular, if we choose $j=n-1$, the residue of the poles associated to a particular eigenvalue $\gamma=\lambda_{i}$ is $1 / n N$.

However, we have to take into account that classical saddle points for the eigenvalues associated to fractional branes $R_{\alpha}^{+}$come located also at $q x, q^{2} x, \ldots q^{r-1} x$ where $r$ is the dimension of the associated representation. And there is a conjugate representation $R^{-} \alpha$ that appears as well for the other set of eigenvalues $q^{r} x, \ldots q^{n-1} x$.

So if we want to count how many eigenvalues $N_{\alpha}$ in the matrix model are located around a classical saddle point which has an eigenvalue located at $x_{0}$, we want to do the following contour integral

$$
\frac{1}{2 \pi i} \oint_{C} R_{n-1}\left(\gamma^{n}\right) d \gamma=\frac{N_{\alpha}^{+}-N_{\alpha}^{-}}{n N}\left(1+q^{-1}+\ldots q^{-r+1}\right)
$$

where $C$ is a contour over the eigenvalue plane which contains $x_{0}$. Notice that the above expression only depends on $N_{\alpha}^{+}-N_{\alpha}^{-}$which is the total number of fractional branes that can not be removed away from the singularity classically. If one studies the Klebanov Strassler system, this would count exactly the difference between the ranks of the gauge groups, which is an invariant under the duality cascade.

Giving specific values to $N_{\alpha} / N$ should determine the curve in the matrix model, by asking which curve has the right residues for a given value of $\mu$. 
In the gauge theory, the generalized resolvent $R_{n-1}$ in the matrix model is related to the generalized resolvent for the chiral ring given by

$$
\mathcal{R}_{j}\left(\gamma^{n}\right)=\operatorname{tr}\left(\frac{X^{j} W^{2}}{\gamma^{n}-X^{n}}\right)
$$

The gauge invariant definition of the partial gaugino condensate $S_{i}$ associated to the fractional branes at some conifold singularity should be given by the following expression

$$
S_{\alpha}=n \frac{1}{2 \pi i\left(1+q^{-1}+\ldots q^{-r+1}\right.} \oint_{C} \mathcal{R}_{n-1}\left(\gamma^{n}\right) d \gamma
$$

where the contour is around one of the eigenvalues of $X$ at the given singularity, and $S_{\alpha}$ is the partial gaugino condensate of the full collection of fractional branes at the singularity, once we get to the bottom of the duality cascade of the local conifold. This expression should be compared with the recent setup of Dijkgraaf and Vafa based on supergroups [20], where everything only depends on the "difference of the gaugino condensates". That statement is equivalent to branes in the bulk not contributing to the deformations of the geometry, as seen also in 谏.

From here, again, it should follow that one should be able to determine the deformation of the geometry caused by the fractional branes.

It would also be interesting to determine whether the above computation coincides with calculating periods of the holomorphic three-form of the associated CY geometry. This problem will be left for future study.

\section{Conclusion}

We have seen in this paper that quantum deformed moduli spaces for geometrically engineered theories encode very non-trivial relations in the associated matrix models. The relations can be argued to be consistency conditions for the loop equations of the matrix model, when the loop equations degenerate. Indeed, the constraints are powerful enough to give a curve which solves the matrix model for a generic number of cuts.

It is not clear under what circumstances the approach presented in this paper is valid. The most conservative possibility is that it applies only to those theories that correspond to a geometric realization of branes at singularities. This is suggested because the effects of the probe brane on fractional branes (what one would call the back reaction) cancels. It is very likely that this is tied to the fact that the low energy effective field theory of the probes on the moduli space is essentially $\mathcal{N}=4 \mathrm{SYM}$, and therefore it does not get corrected, nor does it develop a gaugino condensate. To this extent one can argue that their treatment should be essentially classical. This does not prevent the fractional branes from affecting the geometry of the probe, and we see that indeed they do by forcing geometric

transitions. These transitions exactly deform the points in moduli space where the probe brane effective field theory has less supersymmetry.

The method serves to solve the theory for values of $q$ which are roots of unity. Since in the SUSY gauge theory $q$ is in principle a complex variable, one can expect that the 
solution of vevs of chiral operators depends holomorphically on $q$, with possible singularities at some special values of $q$. These singularities should happen exactly when the associated $S U(2)_{q}$ representations constructed in the paper disappear (at roots of unity). For a given rank of the gauge theory $N$, there are only classically a finite number of values of $q$ which can be pathological (those that are $r$-roots of unity with $r \leq N$, or $q=0, \infty$ ). In principle this information should determine the pole structure of the holomorphic observables of the gauge theory as a function of $q$. Since we can in principle solve the theory for roots of unity, one should be able to use analyticity (holomorphy of the supersymmetric gauge theory) to solve the theory for any $q$, and address the issues of multi-cut solutions of the $\mathcal{N}=1^{*}$ gauge theory. These and other related issues are currently under investigation.

\section{Acknowledgements}

I would like to thank F. Chacazo, M. R. Douglas, D. Gross, A. Hashimoto, V. Kazakov, N. Seiberg, M. Staudacher, E. Witten for various discussions related to this subject. I would also like to thank the Rutgers theory group for their hospitality while this work was developed. Research supported in part by DOE grant DE-FG02-90ER40542.

\section{Appendix}

In this appendix we treat the three matrix model of the paper by considering a saddle point for the eigenvalues of the matrix $X$.

The potential of the theory is given by

$$
N \mu^{-1}(\operatorname{tr}(X Y Z-q X Z Y)+\operatorname{tr}(V(X)))
$$

After one diagonalizes $X$, with eigenvalues $\lambda_{i}$ and if one integrates $Y, Z$, one obtains an effective potential for the eigenvalues of $X$ given by

$$
N \mu^{-1}\left(\sum_{i} V\left(\lambda_{i}\right)\right)+\sum_{i<j}\left[-2 \log \left(\lambda_{i}-\lambda_{j}\right)+\log \left(\lambda_{i}-q \lambda_{j}\right)+\log \left(\lambda_{i}-q^{-1} \lambda_{j}\right)\right]
$$

The saddle point equations for the eigenvalues $\lambda$ are given by

$$
V^{\prime}\left(\lambda_{i}\right)+2 \mu N^{-1} \sum_{j \neq i}\left[-\frac{2}{\lambda_{i}-\lambda_{j}}+\frac{1}{\lambda_{i}-q \lambda_{j}}+\frac{1}{\lambda_{i}-q^{-1} \lambda_{j}}\right]
$$

This is effectively a one matrix model for $X$. Notice that in the limit where the eigenvalue distribution becomes continuous, for each cut in the eigenvalue plane associated

to $X$, there are image cuts in the complex plane rotated by $q$ and $q^{-1}$ in the saddle point equation for the eigenvalues. For the $S U(2)_{1}$ representations one has eigenvalues on these images, and these give a picture of the $S U(2)_{q}$ representations. In the case of $q$ a root of unity, there is a bound on the number of images per cut, and this makes the case of a root of unity special. 
Also, one can derive the first quantum equation 3.46 for the case $q^{n}=1$ by multiplying the above saddle point equation by

$$
\frac{\lambda_{i}}{u-\lambda_{i}^{n}}
$$

summing over all the eigenvalues, and dividing by $N$. However, the author has found no systematic way to extend this procedure to derive the other quantum equations from the above saddle point equations.

\section{References}

[1] O. Aharony, N. Dorey and S. P. Kumar, "New modular invariance in the $\mathrm{N}=1^{*}$ theory, operator mixings and supergravity singularities," JHEP 0006, 026 (2000) [arXiv:hep-th/0006008].

[2] D. Berenstein, "On the universality class of the conifold," JHEP 0111, 060 (2001) [arXiv:hep-th/0110184].

[3] D. Berenstein, "Reverse geometric engineering of singularities," JHEP 0204, 052 (2002) [arXiv:hep-th/0201093].

[4] D. Berenstein, "Quantum moduli spaces from matrix models," Phys. Lett. B 552, 255 (2003) [arXiv:hep-th/0210183].

[5] D. Berenstein, V. Jejjala and R. G. Leigh, "Marginal and relevant deformations of $\mathrm{N}=4$ field theories and non-commutative moduli spaces of vacua," Nucl. Phys. B 589, 196 (2000) [arXiv:hep-th/0005087].

[6] D. Berenstein and R. G. Leigh, "Resolution of stringy singularities by non-commutative algebras," JHEP 0106, 030 (2001) [arXiv:hep-th/0105229].

[7] F. Cachazo, M. R. Douglas, N. Seiberg and E. Witten, "Chiral rings and anomalies in supersymmetric gauge theory," JHEP 0212, 071 (2002) [arXiv:hep-th/0211170].

[8] F. Cachazo, N. Seiberg and E. Witten, "Phases of $N=1$ supersymmetric gauge theories and matrices," arXiv:hep-th/0301006.

[9] F. Cachazo and C. Vafa, "N = 1 and N = 2 geometry from fluxes," arXiv:hep-th/0206017.

[10] F. Cachazo, B. Fiol, K. A. Intriligator, S. Katz and C. Vafa, "A geometric unification of dualities," Nucl. Phys. B 628, 3 (2002) [arXiv:hep-th/0110028].

[11] F. Cachazo, S. Katz and C. Vafa, "Geometric transitions and N = 1 quiver theories," arXiv:hep-th/0108120.

[12] F. Cachazo, K. A. Intriligator and C. Vafa, "A large N duality via a geometric transition," Nucl. Phys. B 603, 3 (2001) [arXiv:hep-th/0103067].

[13] L. Chekhov and A. Mironov, "Matrix models vs. Seiberg-Witten/Whitham theories," Phys. Lett. B 552, 293 (2003) [arXiv:hep-th/0209085].

[14] P. Di Francesco, P. Ginsparg and J. Zinn-Justin, "2-D Gravity and random matrices," Phys. Rept. 254, 1 (1995) [arXiv:hep-th/9306153].

[15] R. Dijkgraaf, M. T. Grisaru, C. S. Lam, C. Vafa and D. Zanon, "Perturbative computation of glueball superpotentials," arXiv:hep-th/0211017. 
[16] R. Dijkgraaf, S. Gukov, V. A. Kazakov and C. Vafa, "Perturbative analysis of gauged matrix models," arXiv:hep-th/0210238.

[17] R. Dijkgraaf and C. Vafa, "Matrix models, topological strings, and supersymmetric gauge theories," arXiv:hep-th/0206255.

[18] R. Dijkgraaf and C. Vafa, "On geometry and matrix models," arXiv:hep-th/0207106.

[19] R. Dijkgraaf and C. Vafa, "A perturbative window into non-perturbative physics," arXiv:hep-th/0208048.

[20] R. Dijkgraaf and C. Vafa, "N = 1 supersymmetry, deconstruction, and bosonic gauge theories," arXiv:hep-th/0302011.

[21] R. Donagi and E. Witten, "Supersymmetric Yang-Mills Theory And Integrable Systems," Nucl. Phys. B 460, 299 (1996) [arXiv:hep-th/9510101].

[22] N. Dorey, T. J. Hollowood and S. Prem Kumar, "An exact elliptic superpotential for N = 1* deformations of finite $\mathrm{N}=2$ gauge theories," Nucl. Phys. B 624, 95 (2002) [arXiv:hep-th/0108221].

[23] N. Dorey, T. J. Hollowood, S. Prem Kumar and A. Sinkovics, "Exact superpotentials from matrix models," arXiv:hep-th/0209089.

[24] N. Dorey, T. J. Hollowood, S. P. Kumar and A. Sinkovics, "Massive vacua of N $=1^{*}$ theory and S-duality from matrix models," arXiv:hep-th/0209099.

[25] N. Dorey, T. J. Hollowood and S. P. Kumar, "S-duality of the Leigh-Strassler deformation via matrix models," JHEP 0212, 003 (2002) [arXiv:hep-th/0210239].

[26] M. R. Douglas, "D-branes and discrete torsion," arXiv:hep-th/9807235.

[27] M. R. Douglas and B. Fiol, "D-branes and discrete torsion. II," arXiv:hep-th/9903031.

[28] F. Ferrari, "Quantum parameter space and double scaling limits in $\mathrm{N}=1$ super Yang-Mills theory," arXiv:hep-th/0211069.

[29] F. Ferrari, "Quantum parameter space in super Yang-Mills. II," arXiv:hep-th/0301157.

[30] H. Fuji and Y. Ookouchi, "Comments on effective superpotentials via matrix models," JHEP 0212, 067 (2002) [arXiv:hep-th/0210148].

[31] P. Ginsparg and G. W. Moore, "Lectures On 2-D Gravity And 2-D String Theory," arXiv:hep-th/9304011.

[32] R. Gopakumar, "N = 1 theories and a geometric master field," arXiv:hep-th/0211100.

[33] R. Gopakumar and C. Vafa, "M-theory and topological strings. I," arXiv:hep-th/9809187.

[34] S. Gukov and D. Tong, "D-brane probes of G(2) holonomy manifolds," Phys. Rev. D 66, 087901 (2002) [arXiv:hep-th/0202125].

[35] S. Gukov and D. Tong, "D-brane probes of special holonomy manifolds, and dynamics of N = 1 three-dimensional gauge theories," JHEP 0204, 050 (2002) [arXiv:hep-th/0202126].

[36] T. J. Hollowood, "New results from glueball superpotentials and matrix models: The Leigh-Strassler deformation," arXiv:hep-th/0212065.

[37] H. Itoyama and A. Morozov, "The Dijkgraaf-Vafa prepotential in the context of general Seiberg-Witten theory," arXiv:hep-th/0211245. 
[38] V. A. Kazakov, "Solvable matrix models," arXiv:hep-th/0003064.

[39] V. A. Kazakov, I. K. Kostov and N. A. Nekrasov, "D-particles, matrix integrals and KP hierarchy," Nucl. Phys. B 557, 413 (1999) [arXiv:hep-th/9810035].

[40] I. R. Klebanov and M. J. Strassler, "Supergravity and a confining gauge theory: Duality cascades and chiSB-resolution of naked singularities," JHEP 0008, 052 (2000)

[arXiv:hep-th/0007191].

[41] I. R. Klebanov and E. Witten, "Superconformal field theory on threebranes at a Calabi-Yau singularity," Nucl. Phys. B 536, 199 (1998) [arXiv:hep-th/9807080].

[42] I. K. Kostov, "Exact solution of the six-vertex model on a random lattice," Nucl. Phys. B 575, 513 (2000) [arXiv:hep-th/9911023].

[43] I. K. Kostov, "Exact solution of the three-color problem on a random lattice," Phys. Lett. B 549, 245 (2002) [arXiv:hep-th/0005190].

[44] R. G. Leigh and M. J. Strassler, "Exactly marginal operators and duality in four-dimensional N=1 supersymmetric gauge theory," Nucl. Phys. B 447, 95 (1995) [arXiv:hep-th/9503121].

[45] T. Mansson, "Another Leigh-Strassler deformation through the matrix model," arXiv:hep-th/0302077.

[46] J. Polchinski and M. J. Strassler, "The string dual of a confining four-dimensional gauge theory," arXiv:hep-th/0003136.

[47] N. Seiberg, "Naturalness versus supersymmetric nonrenormalization theorems," Phys. Lett. B 318, 469 (1993) [arXiv:hep-ph/9309335].

[48] N. Seiberg, "Exact results on the space of vacua of four-dimensional SUSY gauge theories," Phys. Rev. D 49, 6857 (1994) [arXiv:hep-th/9402044].

[49] N. Seiberg, "The Power of holomorphy: Exact results in 4-D SUSY field theories," arXiv:hep-th/9408013.

[50] N. Seiberg and E. Witten, "Electric - magnetic duality, monopole condensation, and confinement in N=2 supersymmetric Yang-Mills theory," Nucl. Phys. B 426, 19 (1994) [Erratum-ibid. B 430, 485 (1994)] [arXiv:hep-th/9407087].

[51] M. Staudacher, "Combinatorial solution of the two matrix model," Phys. Lett. B 305 (1993) 332 [arXiv:hep-th/9301038]. 